\title{
Nuovi criteri per la prospezione sismica a rifrazione
}

\author{
c. CONTINI - C. MAINO - M. RANUCCI
}

\section{ESECUZIONE 1)EI RILIEVI}

\section{1. - Principi gencrali.}

I rilievi sismici a rifrazione vengono ora generalmente applicati seguendo il metodo delle "linee ", il quale consiste nel misurare i tempi impiegati dai raggi sismici per trasmettersi da nn punto di scoppio, clie indicheremo con $S$, a una serie di sismografi, che indicheremo con $G_{1}, G_{2}, \ldots$, disposti sulla superficie del sinolo " varie distanze secondo una linea pressochè retta passante per $S$ stesso.

Il rilievo completo di una linea, sufficiente per la ricostruzione tettonica degli strati del sottosuolo, come si sa, si ha soltanto quando per tutta la lunghezza della stessa linea sono note le dromocrone coningate relative a tutti gli strati. Una tale somma di dati evidentemente si prò ottenere soltanto ripetendo le misure per diverse posizioni dello scoppio, in generale per tante coppie di scopji coniugati quanti sono gli strati presenti nel sottosuolo.

Per la prospezione di una zona completamente sconosciuta dal punto di vista sismico, ronviene come regola iniziare il rilievo con due Iinee ortogonali orientate l'mna perpendicolare e l'altra parallela alle direttrici tettoniche principali, quali risultano dagli elementi geologici noti, operando neI modo seguente:

1. - Indichiamo con $A$ e $B$ gli estremi di una linea da rilevare, quella per esempio perpendicolare alle direttrici tettoniche, con $M$ ed $N$ quelli dell'altra linea e con $O$ il punto d'inter'sezione delle due linee; indichiamo inoltre con $\mathrm{C}, D, P$ e $Q$ rispettivamente $\mathrm{i}$ punti all'incirca intermed $\mathrm{i}$ fra $A$ ed $O, O$ e $B, M$ ed $O, O$ ed $N$; quin di dispo- niamo i sismosgrafi secondo on profilo (stendimento, spread) che inizia da un estremo della linea $A B$, per esempio dall'estremo $A$, c quindi registriamo scoppiando successivamente nei punti $A, C, O, D$ e $B$ dello cariche proporzionate alle distanze fra scoppi e sismografi di registrazione;

2. - Spostiamo il profilo dei sismografi lungo la linea verso $C$ adottando come $1^{0}$ e $2^{\circ}$ sismografi quelli che erano rispettivamente il penultimo e l'ultimo del profilo rilevato (sovrapposizione di due sismografi c di un intervallo) e ancora rileviamo gli scoppi in $A, C, O, D$ e $B$ : e cosi di seguito fino a coprire coi sismografi tutta la linea $A B$;

3. - Operando in modo analogo rilieviamo la linea $M N$ scoppiando nei punti $M, P, O, Q$ ed $N$.

1 risultati di tali misure sono in generale sufficienti per darci un panorama molto preciso delle caratteristiche tettoniche della zona in studio e delle possibilità pratiche dei rilievi sismici a rifrazione. In base a questi elementi potremo stabilive le modalità di rilievo che ci permetteranno di arrivare, nel miglior modo e col minor dispendio, alla ricostruzione degli elementi tettonici che parranno utili ai fini della prospezione.

Noi riteniamo cle la pratica ora sovente adottata di risparmiare il rilievo dei profili prossimi agli scoppi quando interessa prevalentemente il rilievo degli orizzonti profondi sia in ogni caso da respingere per le seguenti ragioni:

a) perchè gli impulsi clıe risultano dai sismogrammi possono sempre essere attribuiti a orizzonti diversi, mancando le correlazioni dirette dei profili adiacenti;

b) perchè il rilievo degli orizzonti profondi non può assolntamente esserc separato da quello degli orizzonti soprastanti. 
Con linee di rilievo contorte o notevolmente fuori dell'allineamento dei punti di scoppio i raggi sismici seguono dei percorsi differenti, e sempre incogniti, per i diversi sismografi dei profili di registrazione e l'interpretazione non può che riuscire grossolana, se non addirittura inattendibile. Per le medesime ragioni è da ritenere sconsigliabile l'impiego del sistema di rilievo per " archi ", ossia coi profili dei sismografi disposti secondo archi di cerchio intorno agli scoppi.

Rilevando per linee nel modo sopra descritto, con registrazioni ripetute nei due sensi coniugati e con diverse distanze fra scoppi e profili dei sismografi, si può raggiungere una velocità di rilievo dell'ordine di quella normale per $\mathrm{i}$ rilievi a riflessione di $1 \div 2 \mathrm{~km}$ di linea coperta al giorno, anche per le zone più difficili, con topografia tormentata e con viabilità minima. Di importanza preponderante agli effetti della velocità di rilievo avrà la scelta delle linee e le ubicazioni delle diverse stazioni di scoppio (corrispondenti ai punti $A, C, O$, $D$ e $B$ della linea $A B$ sopra considerata), alle quali si dovranno perforare $\mathrm{i}$ pozzetti per le mine, accessibili dalle perforatrici automontate $o$ soltanto da perforatrici portatili di efficienza molto limitata.

Cogli strumenti moderni di rilievo, i quali possono anche utilizzare nelle registrazioni frequenze dell'ordine dei due cicli per secondo, la quantità di esplosivo necessaria sarà, in condizioni normali, dell'ordine dei $50 \div 100 \mathrm{~kg}$ di.tritolo, o di altro esplosivo di potenza equivalente, per chilometro di linea rilevata.

Dobbiamo osservare a proposito delle onde di bassa frequenza che queste per trasmettersi senza eccessive attenuazioni necessitano di strati con grandi spessori, non inferiori a mezza lunghezza d'onda, e che quindi il massimo dettaglio di rilievo è raggiungibile soltanto utilizzando onde di frequenza alta nelle registrazioni. In pratica possiamo in generale conciliare le esigenze di rilievo con quelle di contenere i costi utilizzando onde molto corte per le piccole distanze fra sismografi e scoppi e utilizzando onde di maggior lunghezza quando al crescere delle distanze le cariche necessarie per avere buone registrazioni divengono eccessive, per esempio maggiori di $5 \div 10 \mathrm{~kg}$, nelle zone più favorevoli.

Vediamo da queste considerazioni come praticamente certi dettagli che si ottengono colle distanze ridotte fra i sismografi e gli scoppi non siano raggiungibili colle grandi distanze e quindi colle grandi profondità le quali possono essere interessate soltanto colle grandi distanze.

\section{2. - Rilievo dei sismogrammi.}

Le stazioni di scoppio devono essere scelte, in generale, secondo i seguenti criteri: 1) come già abbiamo detto, devono essere il più possibile allineate colle linee di rilievo relative; 2) devono essere facilmente accessibili dagli automezzi che devono perforare i pozzetti e devono rifornire l'esplosivo e l'acqua necessari agli scoppi; 3) devono permettere la postazione di molti pozzetti, necessari per i numerosi scoppi che generalmente si richiedono, abbastanza distanziati in modo che per ogni pozzetto il terreno non risulti smosso dagli scoppi dei pozzetti vicini; 4) devono essere in corrispondenza a terreni consolidati, con aerato non molto potente, al fondo delle valli o delle altre eventuali incisioni del terreno ove la tavola dell'acqua freatica è alle profondità minime $e$ in generale l'efficacia degli scoppi è massima.

I pozzetti di scoppio devono essere preferibilmente tubati con tubi del diametro non minore di $4^{\prime \prime} 1 / 2$. Ripetendo gli scoppi nei pozzetti si terrà conto, nello stabilire la potenza delle cariche, dell'infiacchimento progressivo delle esplosioni.

Dovendo scoppiare delle grandi mine sarà in generale consigliabile ripartire le cariche in molti pozzetti, possibilmente disposti secondo uno o più allineamenti perpendicolari alla linea di rilievo in modo che per ogni pozzetto risultino cariche di lunghezza non maggiore di $15 \div 20 \mathrm{~m}$.

Nelle stazioni di scoppio ove lo spazio risultasse insufficiente per la postazione di tutti i pozzetti necessari si può adottare l'accorgimento di sparare prima tutti i pozzetti possibili alle profondità normali, poi inserire dei pozzetti più profondi e sparare a un livello inferiore, e così di seguito. 
Le distanze lungo le linee di rilievo, fra i sismografi e lo scoppio e fra i diversi sismografi dei profili, devono essere misurate direttamente dal topografo, colla precisione dell'ordine di $\pm \mathbf{5}$ metri; per le quote può bastare la stessa precisione.

Le distanze da adottare fra sismografo e sismografo lungo i profili devono essere fissate in base al grado di dettaglio che si vuole conseguire coi rilievi e alle caratteristiche tettoniche della zona: in generale si possono ritenere più che sufficienti le distanze di $100 \div 200$ metri per le piccole distanze degli scoppi e di $200 \div 500$ metri per le maggiori distanze degli scoppi per le quali le possibilità di dettaglio come si sa sono sempre scarse e d'altra parte si ha interesse a coprire coi sismografi la massima lunghezza possibile, date le grandi cariche impiegate.

Gli inizi dei diversi impulsi devono essere chiari e sicuri e devono essere controllati dal rilevatore mediante le correlazioni coi profili adiacenti prima di spostare il profilo o la stazione di scoppio.

Mediante l'impiego di particolari sistemi di controllo del volume dobbiamo sempre eseguire le registrazioni in modo che risultino rilevabili oltre il primo impulso anche i successivi impulsi.

I sismografi di bassa frequenza propria danno delle registrazioni ottime per tutta la banda delle frequenze alce: usando tali sismografi quindi possiamo in generale operare colle frequenze più adatte per ogni distanza scoppio-sismografi.

La precisione possibile coi rilievi non riesce per nulla diminuita se si riduce la velocità della carta a $1 / 2 \div 1 / 3$ di quella comunemente adottata per i rilievi sismici a riflessione.

Particolare cura deve essere posta per eliminare gli accoppiamenti dei sismografi, sia attraverso i cavi di collegamento dei sismografi col registratore sia attraverso i circuiti interni del registratore.

Disponendo di un registratore con tracce in numero doppio di quello dei sismografi dei profili conviene eseguire le registrazioni in due bande con diverse caratteristiche dei filtri e con diverse amplificazioni.

Come regola si deve adottare un'amplificazione costante per i diversi sismografi o, al più, gradualmente crescente dal sismografo più vicino allo scoppio a quello più lontano, anche quando i disturbi per il vento o per altro risultano di intensità diversa per i diversi sismografi.

\section{LETTURA DEI SISMOGRAMMI}

\section{3. - Considerazioni.}

Gli impulsi (treni d'energia) originati da esplosioni artificiali iniziano sempre con un'onda di compressione e quindi, stando alle convenzioni comunemente accettate, sempre con una punta rivolta verso il basso nei sismogrammi.

La prima mezza onda degli impulsi registrata sui sismogrammi è relativa al primo quarto d'onda dell'impulso del terreno. Questo quarto d'onda è corrispondente, in generale, a una lunghezza d'onda maggiore di quella delle onde successive, essendo dovuto a deformazioni al limite dell'elasticità del terreno per le quali certamente non è valida la legge di Hooke: segue che la lunghezza d'onda corrispondente alla prima mezza onda registrata sarà maggiore della metà di quella delle onde successive. Dagli esperimenti pratici risulta variabile, senza nessuna regola apparente, intorno al rapporto di $3 / 4$.

Tenendo conto di questo fatto riesce in pratica agevolato il riconoscimento dei diversi impulsi che si succedono nei sismogrammi e l'individuazione degli inizi degli stessi.

Agli effetti della registrazione risulta la necessità, per ottenere dei buoni inizi, di adottare dei filtri centrati su lunghezze d'onda circa metà di quelle corrispondenti alle onde di energia prevalente che come regola si devono assumere come base per le registrazioni.

In pratica si nota che di frequente, specialmente per le grandi distanze dagli scoppi, l'onda di compressione è preceduta da una piccola inflessione in senso contrario corrispondente a una lunghezza d'onda del medesimo ordine di grandezza.

Gli impulsi, quali figurano sui sismogrammi, sono sempre costituiti da diverse onde con lunghezze pressochè costanti (a parte 
la mezza onda di compressione iniziale e l'eventuale inflessione preliminare) e con ampiezze dapprima rapidamente crescenti e poi decrescenti secondo la nota legge esponenziale.

\section{4. - Rilievo dei tempi.}

Conviene in pratica rilevare i tempi corrispondenti agli inizi degli impulsi i quali costituiscono la fase più facilmente riconoscibile in quanto: a) sono seguiti dalla mezza onda di compressione di lunghezza ridotta, circa $3 / 4$ delle altre successive costituenti gli impulsi, b) danno luogo a un incremento rapido delle ampiezze delle onde, c) non sono soggetti a fenomeni d'interferenza, quando si tratta di primi impulsi, neanche per $i$ tratti in cui si sopravanzano impulsi di velocità diversa.

Le diverse fasi degli impulsi si susseguono ai diversi sismografi secondo sequenze continue fra loro uguali, più o meno regolari a seconda dei distanziamenti dei sismografi di registrazione, delle caratteristiche elastiche e topografiche del terreno, ecc. Avvenendo quindi che per qualche traccia non siano rilevabili direttamente gli inizi si potranno leggere $\mathrm{i}$ tempi in corrispondenza a fasi successive e quindi ridurre agli inizi aggiungendo le distanze in tempo delle sequenze relative, naturalmente quando non saranno presenti diversi impulsi di velocità differenti.

I diversi impulsi, oltrechè da velocità differenti, sono anche caratterizzati da lunghezze d'onda differenti, sebbene variabili sia colle distanze dal punto di scoppio, sia coll'entità delle cariche e colla specie dell'esplosivo usato, sia colle condizioni del pozzetto, colla natura del terreno in cui avviene lo scoppio, colla profondità dello scoppio stesso, ece.

La precisione del rilievo dei tempi degli inizi degli impulsi può variare in pratica entro limiti vastissimi in relazione alle condizioni geometriche ed elastiche degli strati presenti nel sottosuolo, alle cariche scoppiate, alle distanze dei sismografi dai punti di scoppio, alle modalità seguite nelle registrazioni, ecc.

Quando sono individuabili sicuramente gli inizi degli impulsi l'approssimazione può in generale essere contenuta entro $\pm 10 \mathrm{~ms}$, anche per le maggiori lunghezze d'onda che gh strumenti moderni permettono di utilizzare, la quale non è da considerare troppo scarsa al confronto della precisione che dai rilievi a rifrazione possiamo attenderci. Ogni dubbio, però, sugli impulsi cui attribuire i tempi non può essere eliminato se non colla correlazione coi profili adiacenti, naturalmente relativi al medesimo punto di scoppio.

Gli errori che conseguono all'incertezza sulla fase scelta per i riferimenti possono pure essere eliminati soltanto colle correlazioni coi profili adiacenti.

Praticamente conviene riferire i tempi a una origine convenzionale, in generale scelta coincidente colla linea dei decimi di secondo più vicina all'origine vera, questa coincidente coll'interruzione della traccia di registrazione dei tempi (time break), e ridurre successivamente $i$ valori all'origine vera sottraendo da quelli rilevati il valore corrispondente alla distanza che risulta fra le due origini. Chiamaremo questo valore, espresso in millesecondi, col segno positivo o negativo a seconda se l'origine scelta per i riferimenti è anticipata o ritardata rispetto all'origine vera, con "Addendum " e lo denoteremo col simbolo $\Delta$.

\section{RIDUZIONE DEI VALORI OSSERVATI}

\section{5. - Riduzione al piano base.}

Il piano base di riferimento, indicato generalmente con "Piano base" e abbreviato in "Base" oppure in "P.B. ", sarà scelto di regola orizzontale e corrispondente approssimativamente al livello medio della zona di rilievo, unico per tutta la stessa zona.

Le riduzioni verranno fatte considerando i raggi sismici verticali sia al punto di scoppio sia ai diversi sismografi; si calcoleranno quindi applicando le formole:

$$
\begin{aligned}
& R_{p}=R_{s}+R_{g} \\
& R_{\mathrm{c}}=\frac{H_{s}-H_{o}-p_{s}}{V_{s}}+\frac{\pi_{s}-p_{s}}{V_{a}}\left(1-\frac{V_{a}}{V_{c}}\right) \\
& R_{g}=\frac{H_{g}-H_{o}}{V_{s}}+\frac{\pi_{g}}{V_{n}}\left(\begin{array}{r}
V_{a} \\
V
\end{array}\right),
\end{aligned}
$$


essendo:

$H_{o}$ la quota sul livello del mare del piano base adottato, in metri;

$H_{s}$ la quota sul livello del mare del terreno in corrispondenza del pozzetto di scoppio, in metri;

$H_{\text {o }} \quad$ id. id. in corrispondenza al sismografo considerato;

$p_{s}$ profondità della mina nel pozzetto di scoppio, in metri;

$\pi_{s}$ potenza dello strato aerato superficiale in corrispondenza al pozzetto di scoppio. in metri;

$\pi_{g} \quad$ id. id. in corrispondenza al sismografo considerato, in metri;

$V_{s}$ velocità media del terreno per il tratto compreso fra la base dello strato aerato e il piano base, generalmente diversa in corrispondenza allo scoppio e ai sismografi, in metri al secondo;

$V_{a}$ velocità dell'aerato pure generalmente diversa in corrispondenza allo scoppio e ai sismografi, in metri al secondo.

I due addendi $R_{s}$ ed $R_{o}$ corrispondono rispettivamente alle riduzioni relative allo scoppio e ai sismografi. Il secondo termine dell'espressione di $R_{s}$ sarà considerato nullo sempre quando sarà verificata la condizione $p_{s} \geqslant \pi_{s}$.

I valori di $H_{s}$ e $H_{o}$ saramno dati in generale dai rilievi della squadra topografica e quelli di $p_{s}$ dai bollettini dell'artificiere, con tutta la precisione desiderabile. Molto laboriosa invece riescirà di solito la determinazione dei valori di $\pi_{s}, \pi_{s}$, e $V_{a}$.

Il metodo sovente seguito di determinare lo strato aerato in pochi punti, distanziati anche di chilometri, e di interpolare per $i$ punti intermedi in generale non puo dare dei risultati accettabili nelle zone con topografia non molto tranquilla, incise da torrenti rapidi e tortuosi, coperte da detriti recenti e irregolari, quali più o meno si incontrano in tutti $\mathrm{i}$ rilievi a rifrazione. Noi riteniamo che al confronto possa dare dei risultati meno grossolani il metodo di adottare un valore di riduzione per l'aerato costante medio fra quelli corrispondenti alle condizioni estreme, metodo che, oltretutto, comporta una notevole riduzione della mole dei calcoli di elaborazione dei risultati.

Come regola noi proponiamo di porre costantemente nullo il secondo termine dell'espressione di $R_{s}$ e di adottare per il secondo termine dell'espressione di $R_{g}$ il valore metà di quello massimo che risulta dalle misure dirette, nel rilievo delle dromocrone in prossimità degli scoppi e negli eventuali scoppi effettuati per la riflessione. Indicando con $R_{\max }$ tale valore massimo risulta evidentemente l'errore massimo per le riduzioni di circa $1 / 2 R_{\max }$ e l'errore medio, ammesso che possa applicarsi la legge degli errori di Gauss,

$$
\mu= \pm \frac{1}{6} R_{\max }
$$

E da escludere che in pratica possa convenire il rilievo sistematico dell'aerato, dati i costi molto elevati e i vantaggi per la precisione relativamente scarsi: al più è da prevedere un rilievo del genere per le zone ristrette che risulteranno di particolare interesse e per le quali si rorrà, e risulterà possibile, raggiungere un dettagliamento molto spinto.

I valori di $V_{s}$ da adottare nei calcoli potranno normalmente essere determinati con sufficiente approssimazione mediante poche misure dirette coll'ausilio della carta geologica e colla ricognizione diretta accurata del terreno.

Come conseguenza dell'ipotesi ammessa dei raggi verticali risultano in generale delle dromocrone le quali partono da valori negativi dell'asse dei tempi quando il terreno in corrispondenza allo scoppio è più elevato del piano base e partono da punti alti sull'asse dei tempi quando il terreno è meno elevato.

Il valore della riduzione $R_{s}$, dato dalla [2], costante per ogni sismogramma, viene calcolato nella testata dei bollettini di calcolo (v. paragrafo che segue) mentre quelli di $R_{g}$, dati dalla [3], vengono calcolati nelle colonne dello specchio intestate ai diversi sismografi.

Per l'esecuzione pratica dei calcoli di $R_{s}$ e di $R_{g}$ è consigliabile l'uso del semplice regolo: la precisione dei calcoli numerici dev'essere dell'ordine di $\pm 1 \mathrm{~ms}$. 


\section{6. - Altre riduzioni.}

Altre riduzioni da apportare ai valori osservati dei tempi sono quelle dell'" Addendum " di cui già abbiamo detto, dello "Sfasamento ", della "Fase " e dello "Scoppio scentrato $\%$.

Con "Sfasamento " indichiamo l'intervallo di tempo che dobbiamo sottrarre ai tempi delle onde registrate perchè queste risultino in fase colle deformazioni elastiche dalle quali traggono origine. Tale tempo, che denotiamo con $\varphi$, espresso in millisecondi, evidentemente avrà sempre segno positivo e sarà nullo quando si considereranno gli inizi degli impulsi, del primo impulso e dei successivi.

Colla riduzione della "Fase " riduciamo all'inizio dell'impulso i tempi letti in corrispondenza a una fase qualsiasi la quale appare più chiara nelle registrazioni. Adottiamo per tale riduzione il valore $\psi$, espresso in millisecondi, sempre di segno positivo, che risulta dal prodotto della distanza dei punti letti dall'inizio dell'impulso espressa in lunghezze d'onda per il tempo corrispondente a una lunghezza d'onda dell'impulso considerato (ponendo uguale a 1/3 la lunghezza della prima mezza onda, in generale volta verso il basso dei sismogrammi).

Colla riduzione dello "Scoppio scentrato" riduciamo i tempi osservati al "Centro" della stazione di scoppio di riferimento delle distanze dei sismografi: calcoliamo tale riduzione, che indichiamo con $\delta T$, mediante la fornola:

$$
\delta T=\frac{\delta X}{W_{x}},
$$

ove $\delta X$ sia la componente secondo la direzione dei sismografi dello spostamento del pozzetto di scoppio rispetto al centro della stazione, col segno negativo quando lo stesso spostamento è dalla parte dei sismografi e viceversa, e $W_{x}$ sia la velocità apparente dell'impulso considerato, quale risulta dai raggi rifratti alla superficie del suolo in prossimità della stazione di scoppio, uguale per tutti i sismografi; $\delta T$ in millisecondi, $\delta X$ in metri e $W_{x}$ in $\mathrm{m} / \mathrm{s}$.

Tale formola è valida evidentemente soltanto qui di seguito: fra lo spostamento $\delta Y$ del pozzetto nella direzione con disli- normale a quella dei sismografi e la distanza $X$ dei sismografi stessi sia molto piccolo, per esempio tale che

$$
\delta Y<\sqrt{10 X} .
$$

Gli scostamenti $\delta Y$ notevoli sono da sconsigliare anche per evitare di rilevare raggi con percorsi differenti secondo quanto abbiamo detto al par. 1.

Riportiamo i valori di queste riduzioni, costanti per uno stesso sismogramma e per uno stesso impulso, nella testata dei moduli di calcolo incolonnati in modo che sia agevole farne la somma $R_{s c}=\Delta+\varphi+$ $+\psi+R_{s}+\delta T$.

Nello spazio riservato alle "Osservazioni " dello stesso modulo si potranno mettere in evidenza le caratteristiche topografiche del terreno lungo i profili dei sismografi, le presumibili variazioni della potenza dell'aerato, le condizioni del tempo durante le registrazioni, le caratteristiche peculiari degli impulsi considerati e tutti gli altri elementi che in qualche modo possono tornare utili nell'interpretazione geologica dei risultati.

\section{PRINCIPI PER L'INTERPRETAZIONE GEOLOGICA}

\section{7. - Impostazione dei calcoli.}

Non esiste, nè noi siamo in grado di approntare, nessun metodo d'interpretazione dei risultati dei rilievi sismici a rifrazione il quale permetta la ricostruzione diretta e univoca delle configurazioni tettoniche del suolo.

In generale, quindi, non possiamo fare altro, per l'interpretazione dei risultati, che seguire il vecchio procedimento di ammettere delle particolari configurazioni, di calcolare le dromocrone che in conseguenza si otterrebbero e modificare successivamente le configurazioni in modo da rendere concordanti le dromocrone calcolate colle osservate entro limiti accettabili, conformi all'approssimazione generale dei rilievi.

Come base di tutti i calcoli si assumeranno, di regola, i valori ridotti al piano base, indicati con $T_{r}$ nel modulo di calcolo riportato al paragrafo precedente: sarà opportuno però, nelle zone di topografia tormentata, 
velli che sorpassano i 50 metri, perfezionare l'interpretazione considerando i valori non ridotti della quota, segnati con $T_{c}$ nello stesso modulo di calcolo, e tenere conto delle quote reali dei punti d'osservazione sul terreno.

Per quest'ultimo perfezionamento sarà anche opportuno eseguire la compensazione delle maglie considerando le sezioni normali delle superfici di rifrazione anzichè le sezioni verticali, come in generale si tende a fare nei primi calcoli d'interpretazione.

Vedremo in seguito come in pratica si potrà tenere conto dell'inclinazione delle sezioni: ciò anche al fine della costruzione delle planimetrie le quali, evidentemente, devono in definitiva concludere ogni interpretazione.

Converrà, sempre per agevolare $\mathbf{i}$ calcoli, riportare sovrapposte sui disegni d'interpretazione le dromocrone relative ai tempi ridotti al piano base $T_{r}$ e ai tempi ridotti alla superficie reale del terreno $T_{\iota}$, segnando per esempio i valori di $T$, con circoletti e quelli di $T$, con triangoletti: cosi operando raggiungeremo anche il vantaggio di poter in ogni momento valutare, dalla differenza delle due curve, l'entità della riduzione topografica e quindi renderci conto dell'approssimazione conseguibile nei calcoli.

\section{8. - Ottica dei raggi simici.}

Noi riteniamo che fra i diversi artifici escogitati per rendere rapido il tracciamento dei raggi rifratti secondo la legge di Descartes, al contatto dei diversi mezzi di trasmissione, siano da segnalare il rapportatore di Shoeffler e Diemer (1955) e il nostro regolo che riportiamo nella fig. 1 , entrambi di costruzione semplicissima.
Questo nostro regolo è costituito semplicemente da una striscia di celluloide, dello spessore di mezzo millimetro, trasparente, di forma rettangolare, con incise le linee corrispondenti agli angoli $j$, rispetto a un lato minore, i cui seni hanno i valori di $0,20,0,21,0,22, \ldots 0,99,1,00$.

Il regolo, come è facile comprendere, dà direttamente le direzioni corrispondenti agli angoli limiti di rifrazione $j$, ossia gli angoli dei raggi incidenti, sulle superfici di discontinuità dei mezzi di trasmissione delle onde, i quali danno luogo ad angoli di rifrazione di $90^{\circ}$ (angoli misurati rispetto alle normali alle superfici di discontinuità nei punti d'incidenza). Col regolo si possono tracciare direttamente i raggi incidenti se sul disegno faciamo coincidere le linee del reticolo, cui corrispondono i dati valori del rapporto delle velocità, coi raggi rifratti e contemporaneamente facciamo passare uno dei lati lunghi per i punti dati dei raggi incidenti. Essendo trasparente il regolo può servire per i raggi di tutti e quattro $i$ quadranti.

In effetto però il nostro regolo ha un uso molto più generale. Per esempio:

a) dato il raggio rifratto nel punto $R$ della superficie $\varsigma$ di discontinuità secondo l'angolo $r$ qualunque , $r<90^{\circ}$, per tracciare il raggio incidente rileviamo la linea che forma l'angolo $r$ colla $\varsigma$, moltiplichiamo il valore relativo per il rapporto delle velocità dei mezzi a contatto e quindi tracciamo da $R$ il raggio corrispondente al valore ottenuto;

b) viceversa, dato il raggio incidente secondo l'angolo $i$ qualunque, $i<j$, per tracciare il raggio rifratto rileviamo la linea che forma l'angolo $i$ colla $\varsigma$, dividiamo il rapporto delle velocità per il valore relativo alla linea e quindi da $R$ tracciamo il

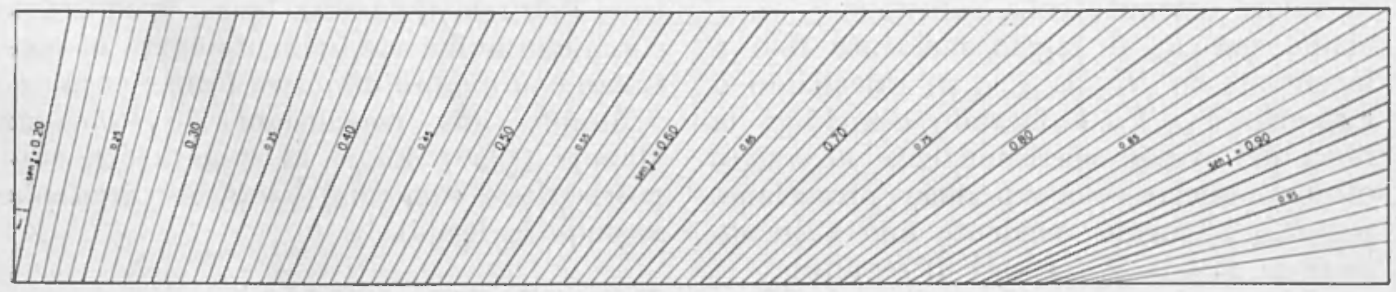

Fig. 1 - Regolo per il tracciamento dei raggi limiti di rifrazione. 
raggio rifratto corrsspondente al valore risultante;

c) quando il sottosuolo fosse costituito da $n$ mezzi di trasmissione diversi, $M_{1}, M_{2}, \ldots$ $M_{n}$, di velocità $V_{n}, V_{2}, \ldots V_{n}$ rispettiva-
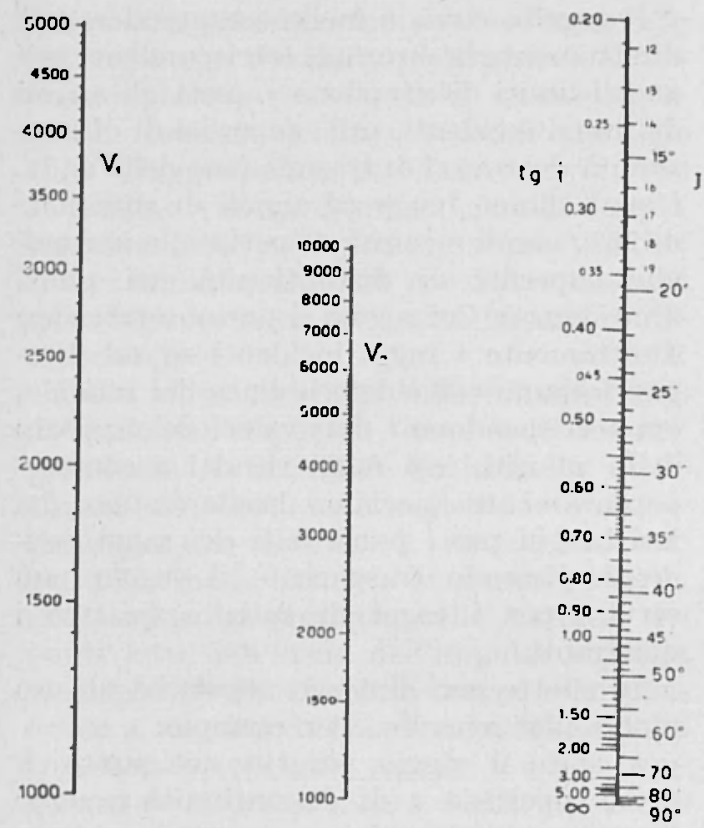

Fig. 2 - Prontuario di calcolo dell' angolo limite di rifrazione $j$ note le velocità $V_{1}$ e $V_{2}$ dei mezzi di trasmissione a contatto.

mente, a contatto secondo le superfici $\varsigma_{2}, \varsigma_{3}$ ... $\varsigma_{n}$, piane e fra loro parallele, dato un raggio rifratto nel punto $R_{n}$ della superficie $\varsigma_{n}$ secondo l'angolo limite $r=90^{\circ}$, determiniamo i punti $R_{n-1}, \ldots R_{2}$ sulle altre superfici di discontinuità nel seguente modo: a) calcoliamo il rapporto $V_{n-1} / V_{n}$ e facendo coincidere la linea del regolo corrispondente al valore ottenuto colla superficie $\varsigma_{n}$ tracciamo per $R_{n}$ il raggio incidente fino a incontrare in $R_{n_{1}}$ la $\varsigma_{n-1}, \beta$ ) calcoliamo il rapporto $V_{n-2} / V_{n}$ e facendo coincidere la linea del regolo corrispondente al valore ottenuto ancora colla superficie $\varsigma_{n}$ tracciamo per $R_{n-1}$ il raggio incidente fino a incontrare in $R_{n-2}$ la $\varsigma_{n-2}$, e così di seguito;

d) nelle stesse condizioni ma colle superfici $\varsigma_{2}, \varsigma_{3}, \ldots \varsigma_{n}$ non fra loro parallele, trac- ciato il raggio nel mezzo $M_{n-1}$, si determinerà il percorso nel mezzo $M_{n-2}$ e negli altri mezzi applicando successivamente quanto indicato in $a$ ).

Nella fig. 2 riportiamo un prontuario di facile uso per il calcolo dei valori dell'angolo limite di rifrazione $j$, noti i valori delle velocità dei mezzi a contatto. I valori di $\mathrm{tg} \mathrm{j}$, segnati accanto a quelli di $j$, possono tornare utili per il tracciamento dei raggi quando si utilizzi la carta millimetrata per i disegni.

\section{9. - La diffrazione.}

In corrispondenza alle discontinuità dei mezzi di trasmissione le onde sismiche danno luogo a fenomeni complessi che soltanto in parte obbediscono alle regole dell'ottica geometrica, i quali vengono genericamente indicati con fenomeni di diffrazione (Contini 1953).

Per quanto riguarda i nostri rilievi sono da considerare le conseguenze di tali fenomeni, particolarmente importanti, sulle leggi della trasmissione dell'energia secondo l'angolo limite di rifrazione e sulla distribuzione dei raggi in corrispondenza alle discontinuità dei mezzi di trasmissione.

Dati due mezzi di trasmissione $M_{1}$ ed $M_{2}$, con velocità $V_{1}$ e $V_{2}$, a contatto secondo una superficie 5 , e la sorgente dei sismi $S$ nel mezzo $M_{1}$, i raggi rifratti da $M_{1}$ ad $M_{2}$ hanno l'energia

$e=\frac{e_{o}}{h^{2}}\left(\frac{V_{1}}{V_{2}}\right) \cos r \sqrt{1-\left(-\frac{V_{1}}{V_{2}}\right)^{2} \operatorname{sen}^{2} r}$,

essendo $h$ la distanza di $S$ dal piano tangente alla $\varsigma$ per il punto di rifrazione $R$ ed $e_{0}$ l'energia dei raggi alla distanza unitaria da $S$ nel mezzo $M_{1}$.

Come si vede, secondo l'ottica geometrica l'energia trasmessa lungo la superficie $\varsigma$ sarebbe nulla: ciò evidentemente in contrasto con quanto dimostra l'esperienza.

Stando alle conclusioni di Romagna Manoja (1956), lungo la superficie $\varsigma$, supposta piana, l'energia dei raggi varierebbe secondo la legge

$$
e_{*}=e_{n} \frac{k \mid \lambda}{x(x-\delta)^{3 / 2}},
$$




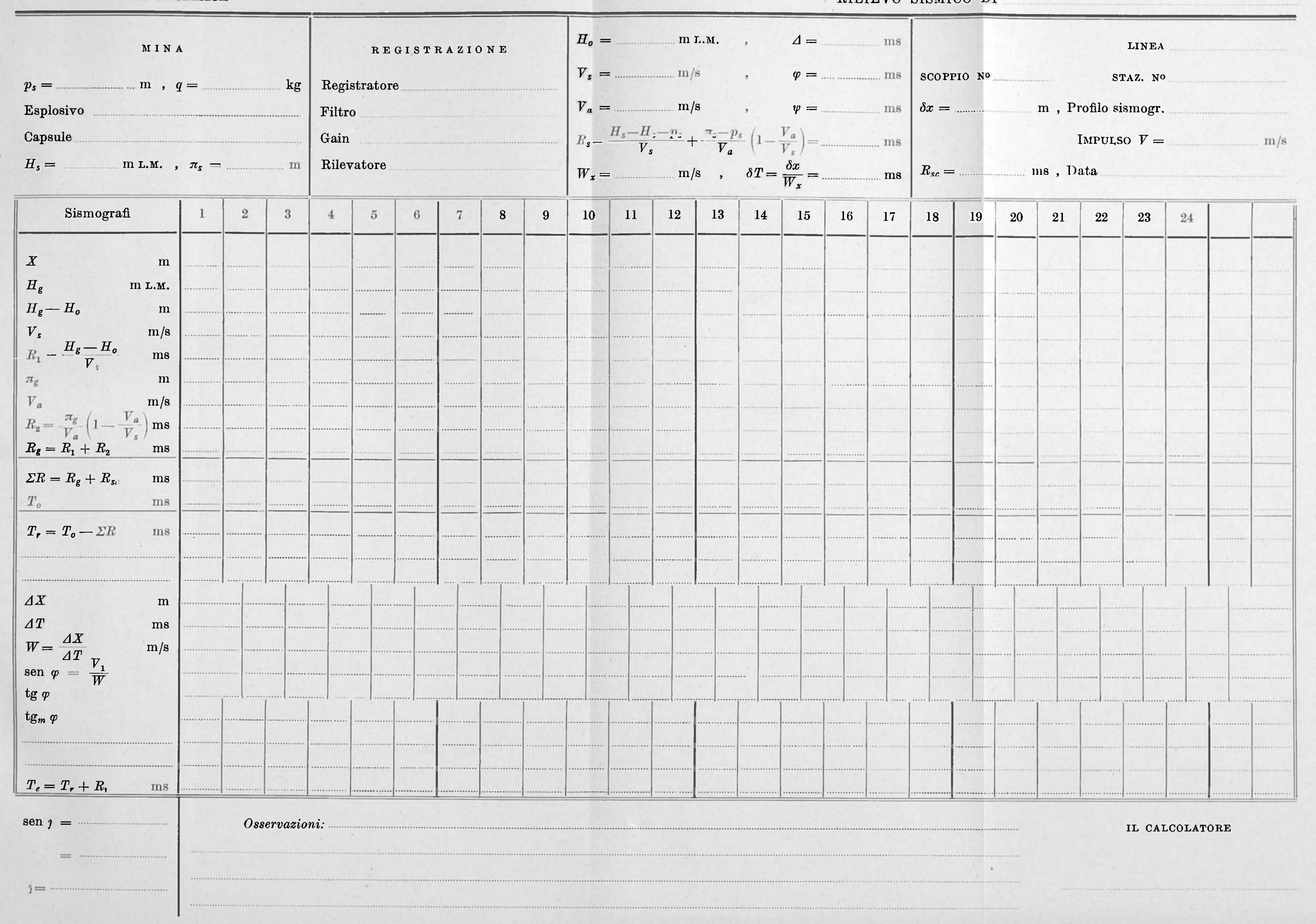


essendo $\delta$ una lunghezza costante da determinarsi, compresa nei limiti $0<\delta<h$ tg $j$, $k$ una costante, $\lambda$ la lunghezza delle onde sismiche ed $x$ le distanze misurate a partire dal punto $S^{\prime}$ di proiezione ortogonale di $S$ su $s$.

Le [4] e [5] possono essere verificate evidentemente soltanto alla condizione che i raggi in $M_{2}$ siano curvi, sebbene con curvature molto piccole, tali da non riescire sensibili nei rilievi pratici.

Ciò posto, riesce facile farci un'idea delle influenze sull'energia dei raggi rifratti alla superficie che possono aversi dalle ondulazioni della superficie $\varsigma$ : influenze, naturalmente, in senso positivo per le inflessioni di 5 verso il basso e negativo per le inflessioni verso l'alto. E da notare clie in generale tali influenze risultano opposte a quelle conseguenti alla convergenza e divergenza dei raggi rifratti dalle superfici curve.

Risulta dalla [5] che le onde lunghe sarebbero consigliabili nei rilievi delle linee molto lunghe, non solo per la loro maggior persistenza in confronto a quelle corte, ma anche perchè danno luogo alla rifrazione verso la superficie del suolo di una maggior percentuale di energia.

Coi fenomeni d'interferenza, che sempre accompagnano quelli della diffrazione, possiamo spiegare come in corrispondenza alle rapide inflessioni degli strati, date le frequenze e le ampiezze variabili delle onde costituenti gli impulsi, si abbiano dei fronti d'onda variabili col tempo e quindi risultino sui sismogrammi delle sequenze che si deformano progressivamente e gradualmente col tempo: il fenomeno naturalmente sarà più o meno appariscente a seconda della lunghezza delle onde registrate.

Per determinare le dromocrone degli strati in corrispondenza alle discontinuità $\mathrm{e}$ alle forti inclinazioni, superiori all'angolo limite $j$, in generale si applicano i principi della brachistocrona, ossia dei tempi minimi, per ogni elemento degli strati e per l'insieme dei diversi elementi. Nel caso, per esempio, di una faglia verticale i tempi successivi a quelli dei punti di discontinuità delle superfici degli strati saranno relativi ai raggi che gli stessi punti irradiano a stella, conformemente ai principi di Huyghens: ciò fino a tanto che risulteranno minori dei tempi relativi ai raggi clie si trasmettono secondo il normale meccanismo della rifrazione dai lembi degli strati oltre la faglia.

\section{STRATI PIANI \\ CON VELOCITA COSTANTE}

\section{0. - Generalità.}

Come si sa, la velocità di trasmissione delle onde sismiche dei terreni varia notevolmente colle diverse specie delle rocce e per ogni specie è funzione della compattezza e del grado di costipazione raggiunti e quindi delle pressioni clie si esercitarono dopo la messa in posto, del tempo d'azione delle stesse, delle temperature che si manifestarono, dei fluidi contenuti, della pressione degli stessi, ecc.

L'azione di questi fattori non potè che estrinsecarsi in generale con efficacia crescente colla profondità: di regola quindi avremo un aumento della velocità di trasmissione delle onde colla profondità e coll'età dei terreni.

In pratica però avviene non di rado, per rocce di particolare costituzione e per potenze limitate, di trovare delle velocità variabili secondo la profondità con incrementi molto ridotti, tali da riuscire coperti dall'approssimazione limitata conseguibile coi rilievi e dalle normali eterogeneità delle rocce stratificate: in tali condizioni converrà senz'altro adottare dei valori costanti della velocità.

Come eseguire i calcoli per la determinazione della legge della velocità non è possibile precisare in generale, dato che $\mathrm{i}$ valori di osservazione sono influenzati in modo molto variabile dalle configurazioni geometriche delle superfici di contat to delle diverse specie di rocce costituenti il sottosuolo, le quali di norma costituiscono l'oggetto dei rilievi e quindi o sono sconosciute o sono note soltanto nelle loro grandi linee. Ora noi possiamo soltanto consigliare:

1) di adottare per i primi calcoli delle formole provvisorie per le velocità dei terreni di rilievo e perfezionare progressivamente le stesse formole in modo da soddisfare nel miglior modo i dati che via via vengono acquisiti coi rilievi; 
2) di ammettere le variazioni laterali delle velocità con molta prudenza, se non diffidenza, per non correre il rischio di mascherare in tale modo delle particolarità tettoniche, anche di notevolissimo interesse pratico.

Pel caso che si assuma per i diversi strati dei valori costanti della velocità, il quale è certamente il più semplice che in pratica si possa presentare, aggiungiamo questi altri consigli:

I) basare i calcoli di preferenza sulle dromocrone che nei due sensi coniugati risultano rettilinee o, perlomeno, con curvature tali per cui gli eventuali errori di calcolo delle inclinazioni degli strati relativi nei punti di rifrazione risultano sicuramente minori dei limiti che conseguono dall'approssimazione generale delle misure;

II) per lo strato superficiale utilizzare i valori relativi ai sismografi il più possibile lontani dai punti di scoppio per i quali sono sempre da supporre minimi gli effetti negativi delle eterogeneità dei terreni in prossimità della superficie del suolo nonchè dalle asperità topografiche in corrispondenza ai punti di scoppio.

Nei paragrafi che seguono, relativi all'ipotesi di un sottosuolo costituito da strati di velocità costante e limitati da superfici piane, i simboli hanno il seguente significato:

$V_{1}, V_{2}, V_{3} \ldots$ velocità di trasmissione delle onde sismiche rispettivamente degli strati primo, secondo, terzo, ... a incominciare dalla superficie del suolo;

$S$ punto origine dei sismi, in generale coincidente coll'intersezione col piano base della verticale per il punto di scoppio;

$G$ punto di ricezione dei sismi, in generale coincidenti coll'intersezione col piano base delle verticali per i punti della superficie del suolo ove sono postati i sismografi di rilievo (geofoni);

$X$ distanze dall'origine dei sismi misurate orizzontalmente, in metri;

$T$ tempi rilevati dai sismogrammi ridotti e riferiti al piano base, supposto orizzontale, in millesecondi;

$\xi_{1}, \zeta_{2}, \xi_{3}, \ldots$ dromocrone relative agli strati $1^{\circ}, 2^{\circ}, 3^{\circ}, \ldots$;

$T_{i 2}, T_{i 3}, \ldots$ tempi intercetti sull'asse dei tempi $(X=O)$ dalle dromocrone $\xi_{2}$, $\xi_{3}, \ldots$ " (intercept time "), in millisecondi;

$\varsigma_{12}, \varsigma_{23}, \ldots$ superfici (piani) di contatto fra il $1^{\circ}$ e il $2^{\circ}$ strato, fra il $2^{\circ}$ e il $3^{\circ}$ strato, ....;

$a_{2}, a_{3}, \ldots$ inclinazioni delle superfici $\varsigma_{12}$, $\varsigma_{23}, \ldots$, , in gradi sessagesimali;

$z_{2 s}, z_{3 s}, \ldots$ profondità delle superfici $\varsigma_{12}, \varsigma_{23}, \ldots$ in corrispondenza all'origine dei sismi $S$, in metri;

$z_{2:}, z_{3 g}, \ldots$ le stesse profondità in corrispondenza ai punti di rilievo dei sismi $G$;

$d_{2 s}, d_{3 s}, \ldots$ distanze delle superfici $\varsigma_{12}$, $\varsigma_{23}, \ldots$ dall'origine dei sismi $S$, in metri;

$d_{2 g}, d_{3 g}, \ldots$ le stesse distanze dai punti di rilievo dei sismi $G$;

$j_{2}, j_{3}, \ldots$ angoli limiti di rifrazione dei raggi incidenti sulle superfici $\varsigma_{12}, \varsigma_{23}, \ldots$, misurati rispetto alle normali alle stesse superfici, in gradi sessagesimali (sen $j_{2}=$ $V_{1} / V_{2}$, sen $j_{3}=V_{2} / V_{3}, \ldots$ );

$W_{2 i t}$, "Wu,$\ldots$ velocità apparenti degli strati $2^{\circ}, 3^{\circ}, \ldots$ quali risultano dalle pendenze delle linee dromocrone, rispetto all'asse dei tempi, nel caso che gli strati si " elevino " (up) nel verso delle distanze crescenti, in metri al secondo;

$W_{2 d}, W_{3 d}, \ldots$ le stesse velocità apparenti nel caso che gli strati si "abbassino" (down) nel verso delle distanze crescenti delle dromocrone;

$W_{2 m}, W_{3 m}, \ldots$ le medie delle stesse velocità quali risultano per i due versi opposti, $W_{2 m}=\left(W_{2 u}+W_{2 d}\right) / 2, W_{3 m}=\left(W_{3 u}+W_{3 d}\right) / 2$, $\ldots$;

$\Delta_{2}, \Delta_{3}, \ldots$ le differenze fra le stesse velocità relative al verso secondo cui gli strati si elevano e al verso opposto, $\Delta_{2}=$ $W_{2 u} \rightarrow W_{2 d}, \Delta_{3}=W_{3 u}-W_{3 d}, \ldots$.

Per comodità sovente indicheremo con " coniugati " gli elementi di rilievo relativi agli stessi punti di rifrazione che risultano scoppiando dalle due parti opposte.

\section{1. - Due strati piani.}

Ammettiamo di avere un sottosuolo costituito da due strati, omogenei ed isotropi, di velocità $V_{2}$ e $V_{2}$, a contatto secondo una superficie $\varsigma_{12}$, piana e continua, di inclinazione $\alpha_{2}$ e inoltre ammettiamo di operare mediante linee disposte secondo la massima pendenza della superficie $\varsigma_{12}$. 
In tale caso l'equazione della dromocrona relativa al primo strato, è, evidentemente, adottando le convenzioni del paragrafo col segno per $\alpha_{2}$ negativo o positivo a seconda se la $\zeta_{12}$ si eleva oppure si abbassa nel verso delle $\mathrm{x}$ crescenti.

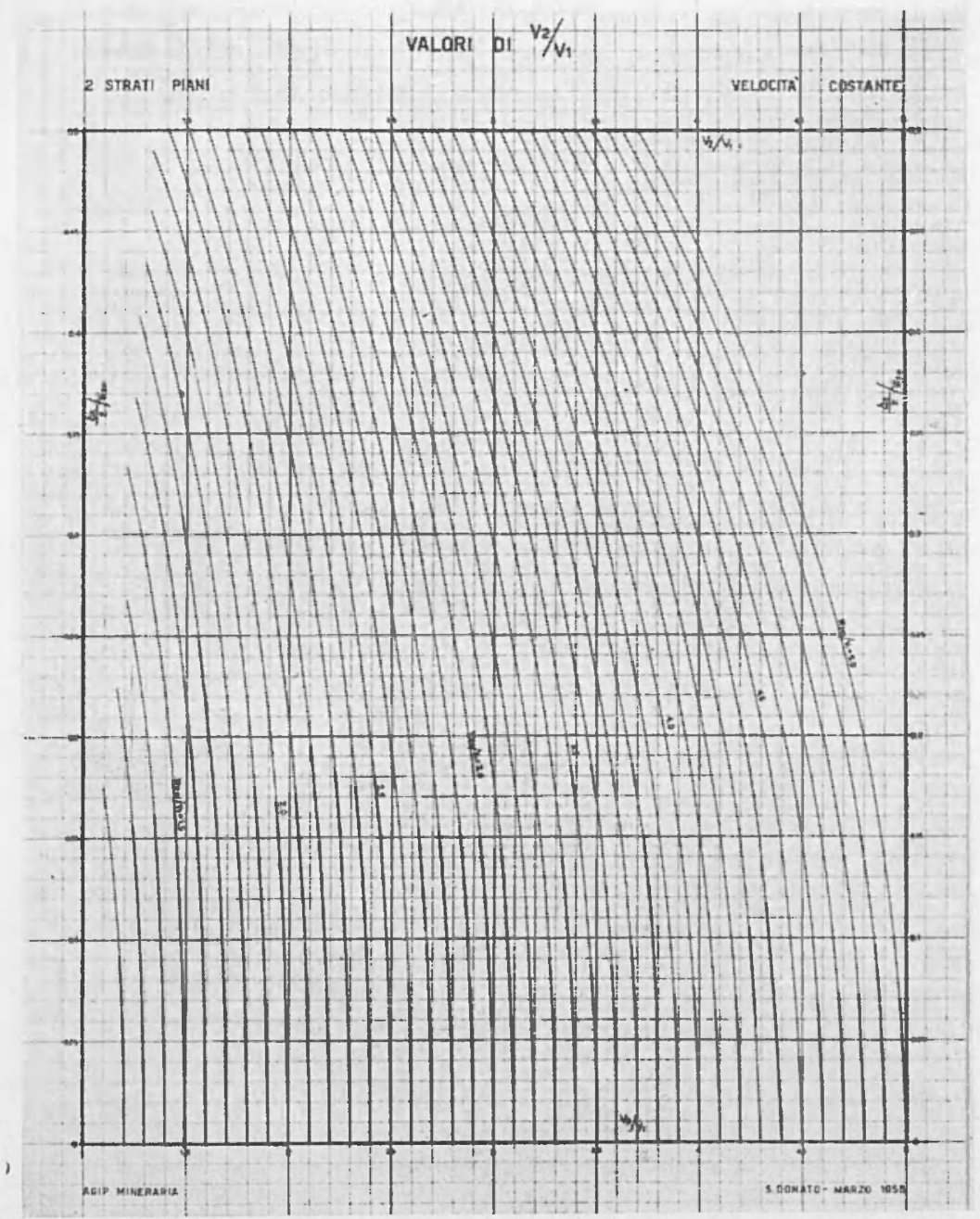

Fig. 3 - Prontuario di calcolo del rapporto $V_{2} / V_{1}$ noti i valori dei rapporti $\Delta_{2} / 2 W_{2 m}$ e $W_{2 m} / V_{1}$.

precedente,

$$
T=\frac{X}{V_{1}} .
$$

L'equazione della dromocrona relativa al secondo strato è invece, fig. 7,

$$
T=\frac{1}{V_{1}}\left\{2 d_{2 s} \cos j_{2}+X \operatorname{sen}\left(j_{2} \mp \alpha_{2}\right) \mid\right.
$$

Le velocità apparenti sono date dalle derivate delle distanze $X$ per rispetto al tempo $T$ : dalla [7] otteniamo allora:

$$
\begin{aligned}
& W_{\text {.. }}-\frac{V_{1}}{\operatorname{sen}\left(j_{2}-a_{2}\right)} \\
& W_{2 d}=\frac{V_{1}}{\operatorname{sen}\left(j_{2}+\alpha_{2}\right)} .
\end{aligned}
$$


Dal sistema formato da queste due equa- Nella fig. 3 riportiamo la riduzione fotozioni otteniamo, con facili trasformazioni, grafica del grafico-prontuario di calcolo del

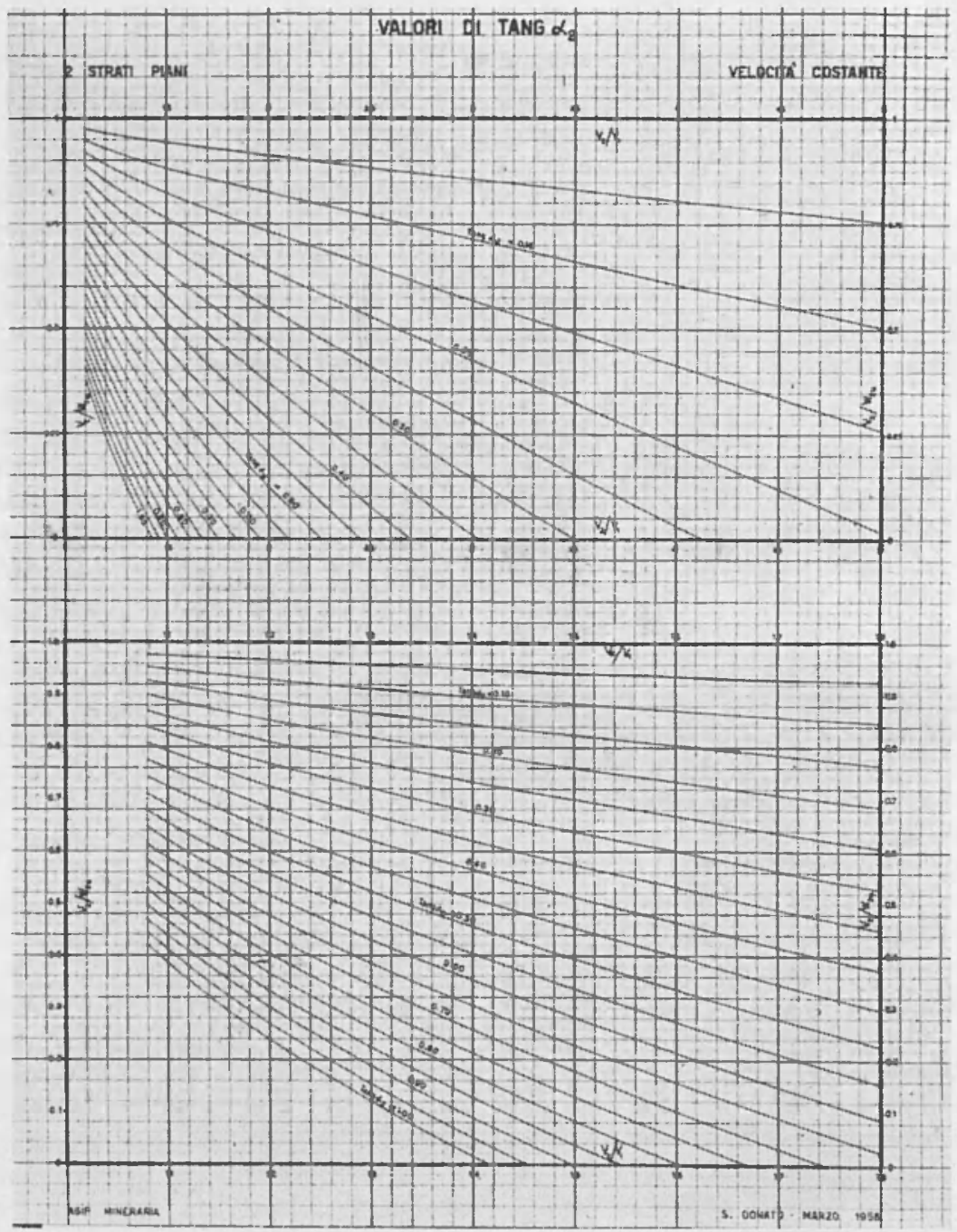

Fig. 4 - Prontuario di calcolo delle pendenze $\operatorname{tg} a_{2}$ noti i valori dei rapporti $\Gamma_{2} / \|_{2 u}$ e $V_{2} / V_{1}$

la formola di calcolo della velocità vera $V_{2}$

$$
2\left(\frac{V_{2}}{-V_{1}}\right)^{2}=A+B^{2}+\sqrt{\left(A+B^{2}\right)^{2}-4 B^{2}}[9]
$$

rapporto $V_{2} / V_{1}$ in funzione dei valori di $\Delta_{2} / 2 W_{2 m}$ e $W_{2 m} / V_{1}$ che abbiamo costruito in base alla [9], molto utile per la pratica.

Posto noto il valore di $\mathrm{V}_{2}$ possiamo deterove

$$
A=1-\left(\frac{\Delta_{2}}{2 W_{2 m}}\right)^{2}, \quad B=\frac{W_{2 m}}{V_{1}} \mathrm{~A} .
$$
minare le pendenze $\operatorname{tg} \alpha_{2}$ dalle singole dromocrone mediante le formole che si ricavano pure dalle [8] 


$$
\operatorname{tg} \alpha_{2}=\frac{\sqrt{\left.\left(\frac{V_{2}}{V_{1}}\right)^{2}-1-\frac{V_{2}}{W_{2 n}}\right]}}{\left(\frac{V_{2}}{V_{1}}\right)^{2}-\left(\frac{V_{2}}{\left(\frac{V_{2}}{V_{2}}\right)^{2}-\left(\frac{V_{2}}{W_{2 u}}\right)^{2}}\right.}
$$

la prima relativa al caso clie lo strato si elevi nel verso delle $X$ crescenti e la seconda relativa al caso inverso.

Nelle figure 4 e 5 riportiamo le riduzioni fotografiche dei grafici-prontuari di calcolo delle pendenze $\operatorname{tg} \alpha_{2}$ approntati in base alle [10].

Determiniamo la distanza $d_{2 s}$ della superficie $\varsigma_{12}$ dal punto di scoppio $S$ risolvendo

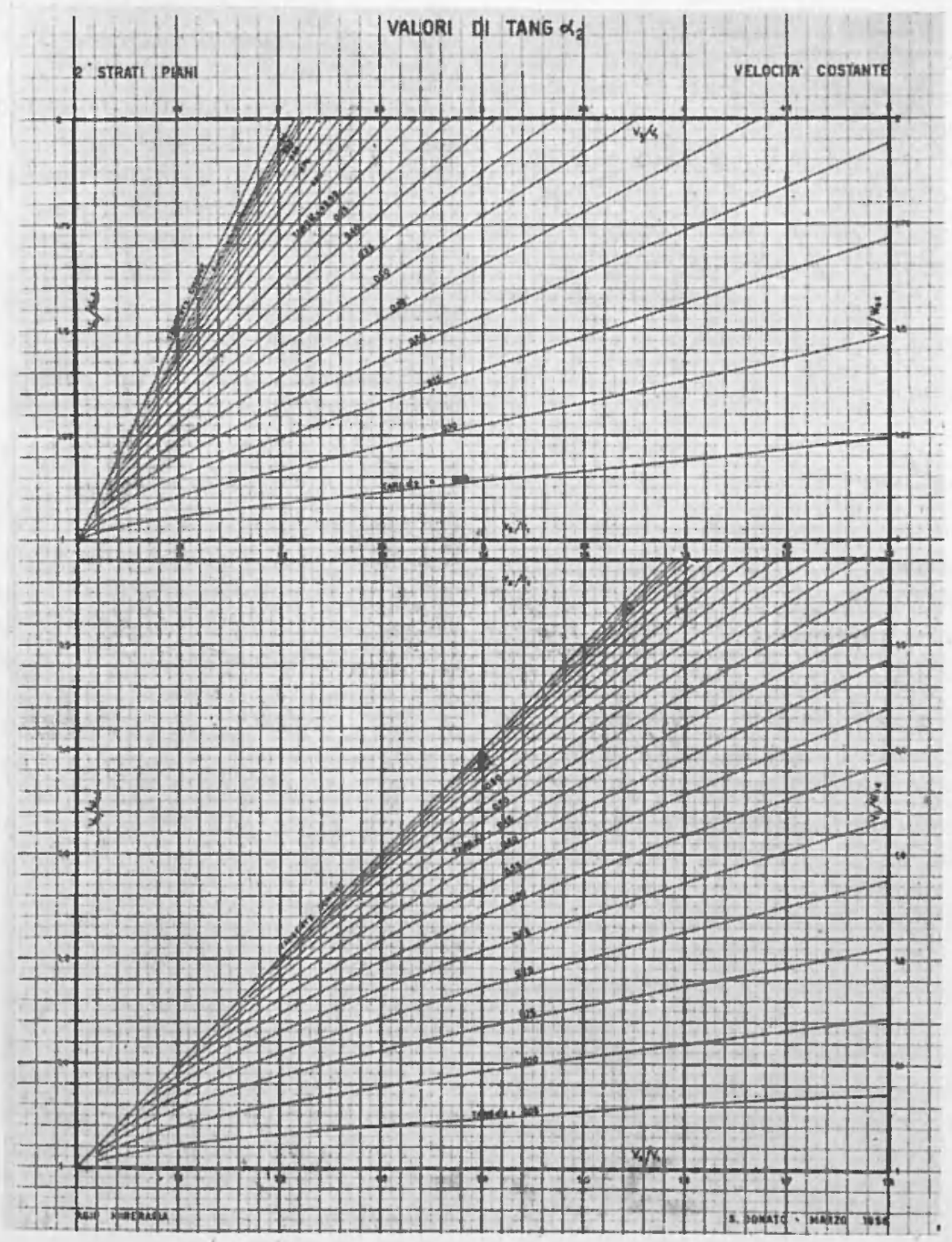

Fig. 5 - Prontuario di calcolo delle pendenze $\operatorname{tg} \alpha_{2}$ noti i valori dei rapporti $F_{2} / W_{2 d}$ e $V_{2} / V_{1}$ 
le equazioni che risultano dalla [7] posto $X=0$

$$
d_{2 s}=\frac{\frac{V_{2}}{V_{1}}}{\sqrt{\left(\frac{V_{2}}{V_{1}}\right)^{2}-1}} V_{1} \frac{T_{i 2}}{2},
$$

essendo $T_{22}$ il tempo intercetto.

Le formole sopra trovate sono valide, in effetto, qualunque sia l'orientamento, rispetto alla direzione del secondo strato,

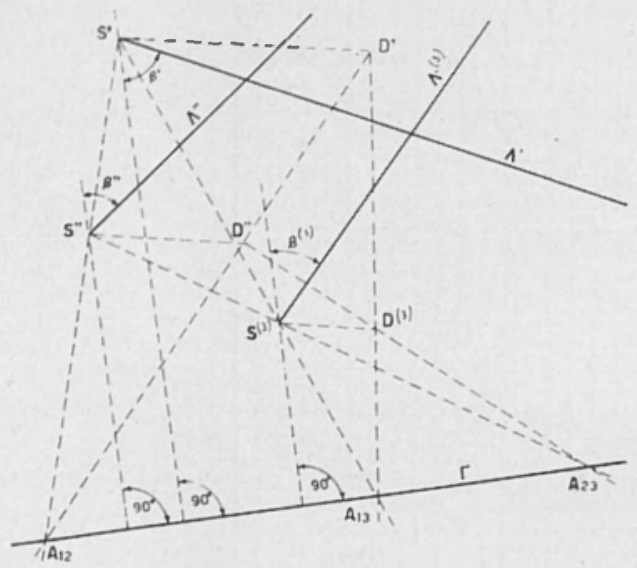

Fig. 6 - Determinazione della direzione dello strato noti i tempi intercetti di tre stazioni di scoppio qualsiasi.

della "linea " di rilievo cui sono riferite le dromocrone considerate: naturalmente alla condizione che le pendenze $\operatorname{tg} \alpha_{2}$ siano riferite al piano normale alla superficie $\varsigma_{12}$, limite superiore del secondo strato, contenente la " linea " di rilievo.

Supposte note le pendenze $\operatorname{tg} \alpha_{2}^{\prime}$ e $\operatorname{tg} \alpha^{\prime \prime}{ }_{2}$ relative a due linee qualunque $\Lambda^{\prime}$ e $\Lambda^{\prime \prime}$ incrociantisi nel punto $I_{2}$, determiniamo la direzione di massima pendenza e la massima pendenza stessa mediante la seguente costruzione che si ricava facilmente da quella nota della composizione vettoriale delle pendenze:

a) portiamo sulle tracce di $\Lambda^{\prime}$ e $\Lambda^{\prime \prime}$, a partire da $I_{2}$, i segmenti $I_{2} Q^{\prime}{ }_{2}$ e $I_{2} Q^{\prime \prime}{ }_{2}$ proporzionali ai valori di $\operatorname{sen} \alpha^{\prime}{ }_{2}$ e $\operatorname{sen} \alpha_{2}^{\prime \prime}$ che si ricavano da quelli noti delle pendenze mediante le normali tabelle dei valori trigonometrici; b) tracciamo da $Q^{\prime}{ }_{2}$ la perpendicolare alla traccia di $\Lambda^{\prime}$ e da $Q^{\prime \prime}{ }_{2}$ la perpendicolare alla traccia di $\Lambda^{\prime \prime}$ e congiungiamo $I_{2}$ col punto $Q_{2}$ d'intersezione delle perpendicolari: il segmento $I_{2} Q_{2}$ rappresenta in direzione la linea di massima pendenza e in grandezza il seno dell'angolo di massima inclinazione $\operatorname{sen} a_{2}$.

Possiamo facilmente vedere che per le piccole pendenze, minori del $30 \%$, in effetto possono essere utilizzati, per il calcolo delle pendenze totali degli strati, indifferentemente $\mathrm{i}$ vettori tangente e $\mathrm{i}$ vettori seno, date le differenze minime dei valori relativi, sempre minori del centesimo.

Consideriamo infine il caso che il rilievo sia costituito da tante linee $\Lambda^{\prime}, \Lambda^{\prime \prime}, \Lambda^{(3)}, \ldots$ disposte secondo le regole del caso, rilevate in una sola direzione: casi del genere possono aversi in zone di topografia molto difficile prive di strade e quando si vogliono utilizzare per gli scoppi dei corsi d'acqua, laghi o il mare.

Possiamo operare nel modo seguente, riferendoci alle indicazioni della fig. 6:

$A$ ) sulla planimetria delle linee di rilievo, dalle stazioni di scoppio $S^{\prime}, S^{\prime \prime}, S^{(3)}, \ldots$ tracciamo tante parallele secondo una direzione qualsiasi e a partire dalle stazioni di scoppio stesse, portiamo, secondo un verso prefissato, dei segmenti proporzionali ai tempi intercetti delle linee relative $T^{\prime}{ }_{22}$, $T^{\prime \prime}{ }_{i 2}, T^{(3)}{ }_{i 2}, \ldots$. rispettivamente in $S^{\prime} D^{\prime}$, $S^{\prime \prime} D^{\prime \prime}, S^{(3)} D^{(3)}, \ldots \ldots$ determiniamo i punti d'intersezione $A_{12}, A_{13}, \ldots . \mathrm{A}_{23}, A_{24}, \ldots$. fra le linee $S^{\prime} S^{\prime \prime}$ e $D^{\prime} D^{\prime \prime}, S^{\prime} S^{(3)}$ e $D^{\prime} D^{(3)}, \ldots$ $S^{\prime \prime} S^{(3)}$ e $D^{\prime \prime} D^{(3)}, S^{\prime \prime} S^{(4)}$ e $D^{\prime \prime} D^{(4)}, \ldots \ldots$ e quindi tracciamo la retta $\Gamma$ che congiunge i punti $A_{12}, A_{13}, \ldots A_{23}, A_{24}, \ldots$ per la quale gli scostamenti risultano minimi, secondo i principi della teoria dei minimi quadrati: tale linea è la traccia del piano limite superiore dello strato $\varsigma_{12}$ sul P.B.;

$B)$ dalle [8] ricaviamo l'espressione di $\operatorname{sen} \alpha_{2}$ come funzione dei rapporti $V_{1} / V_{2}$ e di $V_{1} / W_{2}$, essendo con $W_{2}$ indicata genericamente la velocità apparente risultante dalla dromocrona del secondo strato,

$\operatorname{sen} \alpha_{2}=\mp \frac{V_{1}}{W_{3}} / \sqrt{1-\left(\frac{V_{1}}{Y_{2}}\right)^{2}} \pm \frac{V_{1}}{V_{2}} \sqrt{1-\left(\frac{V_{1}}{W_{2}}\right)^{2}}$ 
coi segni dei due termini sempre contrari, i primi quando $W_{2}>V_{2}$ e i secondi quando $W_{2}<V_{2}$, quindi misuriamo gli angoli acuti $\beta^{\prime}, \beta^{\prime \prime}, \beta^{(3)}, \ldots$ formati dalle linee $\Lambda^{\prime}$, $\Lambda^{\prime \prime}, \Lambda^{(3)}, \ldots$ colla direzione di massima pendenza (normale alla traccia $\Gamma$ ) e poniamo le uguaglianze

$$
\frac{\operatorname{sen} \alpha_{2}^{\prime}}{\cos \beta^{\prime}}-\frac{\operatorname{sen} \alpha_{2}^{\prime \prime}}{\cos \beta^{\prime \prime}}-\frac{\operatorname{sen} \alpha_{a}^{(\bar{a})}}{\cos \beta^{(3)}}=\ldots \ldots,
$$

ove $\operatorname{sen} \alpha_{2}^{\prime}, \operatorname{sen} \alpha_{2}^{\prime \prime}, \operatorname{sen} \alpha_{2,}^{(3)}, \ldots$ siano $\mathrm{i}$ valori della $[12]$ relativi alle linee $\Lambda^{\prime}, \Lambda^{\prime \prime}$, $\Lambda^{(3)}, \ldots$ rispettivamente: se quindi poniamo

$$
\begin{aligned}
& \Psi^{\prime}=\frac{1}{\cos \beta^{\prime}} \frac{V_{1}}{W_{2}{ }^{\prime}}, \\
& \Psi^{\prime \prime}=\frac{1}{\cos \beta^{\prime \prime}} \frac{V_{1}}{W_{2}^{\prime \prime}}, \\
& \ldots \ldots \ldots \ldots \ldots \ldots \ldots \\
& \Phi^{\prime}=\frac{1}{\cos \beta^{\prime}} \sqrt{1-\left(\frac{V_{1}}{W_{2}^{\prime}}\right)^{2}} \\
& \Phi^{\prime \prime}=\frac{1}{\cos \beta^{\prime \prime}} \sqrt{1-\left(\begin{array}{c}
\left.V_{1}\right)^{2} \\
W_{2}^{\prime \prime}
\end{array}\right)^{\prime}} \\
& \ldots \ldots \ldots . \cdots \\
& R_{12}-\frac{\Phi^{\prime}-\Phi^{\prime \prime}}{\Psi^{\prime}-\Psi^{\prime \prime}}, \\
& R_{13}=\frac{\Phi^{\prime}-\Phi^{(3)}}{\Psi^{\prime}-\Psi^{(3)}}, \\
& \ldots \ldots \ldots \ldots \\
& R_{23}-\frac{\Phi^{\prime \prime}-\Phi^{(3)}}{\Psi^{\prime \prime}-\Psi^{(3)}}, \\
& R_{21}=\frac{\Phi^{\prime \prime}-\Phi^{(4)}}{\Psi^{\prime \prime}-\Psi^{(4)}},
\end{aligned}
$$

otteniamo

$$
\frac{V_{2}}{V_{1}}-\stackrel{R}{2}_{m}^{2}+1
$$

ove $R_{n}$ è il valore medio ponderale di $R_{12}$, $R_{n, .} \ldots R_{23}, R_{2}$....;

C) determinato in tale modo il valore più attendibile del rapporto $V_{2} / V_{1}$ determiniamo per ogni linea rilevata la pendenza mediante i grafici delle figure 4 e 5 , calcoliamo $i$ valori corrispondenti del seno, dividiamo per i coseni degli angoli di direzione delle linee corrispondenti $\cos \beta^{\prime}, \cos$ $\beta^{\prime \prime}, \ldots$, e infine facciamo la media ponderale: questa media è evidentemente il valore più attendibile del seno dell'inclinazione totale dello strato.

12. - Tre o più strati piani.

Consideriamo ora il caso di un sottosuolo costituito da $n$ strati omogenei ed isotropi di velocità $V_{1}, V_{2}, \ldots V_{n}$ a contatto secondo le superfici $\varsigma_{12}, \varsigma_{23}, \ldots \ldots \varsigma_{(n-1) n}$ piane e continue di uguale direzione con inclinazioni $a_{2}, \alpha_{3}, \ldots \ldots \alpha_{n}$ e, inoltre, ammettiamo di disporre le linee di rilievo secondo la massima pendenza degli strati.

Operando in modo analogo a quello seguito per i i due strati otteniamo, riferendoci alle indicazioni della fig. 7 , nel caso di tre strati

$$
\begin{aligned}
& W_{3 u}=\frac{V_{1}}{\operatorname{sen}\left(i_{13}^{\prime \prime}-a_{2}\right)}, \quad w_{3 d}=\frac{V_{1}}{\operatorname{sen}\left(i_{13}^{\prime}+a_{2}\right)}, \\
& T_{i 3}=2 \frac{d_{3 s}}{V_{2}} \cos j_{3}+\frac{d_{2 s}}{V_{1}}\left(\cos i_{13}^{\prime}+\cos i_{13}^{\prime \prime}\right) \cdot[15]
\end{aligned}
$$

Da queste equazioni, essendo noti $V_{1}$, $V_{2}, a_{2}, d_{2 s}, W_{3 u}, W_{3 d}$, e $T_{i 3}$, possiamo determinare i valori di $i_{13}^{\prime}$ e $i_{3}^{\prime \prime}$ ponendo

$$
\begin{aligned}
& i_{13}^{\prime \prime}=\operatorname{arcsen} \frac{V_{1}}{\dddot{w}_{3 u}}+a_{2}, \\
& \ddot{i}_{13}=\operatorname{arcsen} \frac{V_{1}}{W_{3 d}}-a_{2} .
\end{aligned}
$$

Noti $i_{13}^{\prime}$ e $i_{13}^{\prime \prime}$ essendo

$r_{13}^{\prime}=\operatorname{arcsen}\left(\frac{V_{2}}{V} \operatorname{sen} \ddot{i}_{13}\right)=j_{3}+\left(\alpha_{3}-\alpha_{2}\right)$,

$r_{13}^{\prime \prime}=\operatorname{arcsen}\left(\frac{V_{2}}{V_{1}^{-}} \operatorname{sen} i_{13}^{\prime \prime}\right)=j_{3}-\left(\alpha_{3}-\alpha_{2}\right)$,

otteniamo i valori del rapporto $V_{2} / V_{3}$ e dell'inclinazione $a_{3}$ ponendo

$$
\begin{gathered}
\frac{V_{2}}{V_{3}}=\operatorname{sen} j_{3}=\operatorname{sen} \frac{1}{2}\left(r_{13}^{\prime}+r_{13}^{\prime \prime}\right) \\
a_{3}=a_{2}+\frac{1}{2}\left(r_{13}^{\prime}-r_{13}^{\prime \prime}\right) .
\end{gathered}
$$

Dall'ultima delle [15] infine otteniamo il valore della distanza $d_{3 s}$ della $\varsigma_{23}$ dal punto $S_{2 d}$ d'intersezione colla $\varsigma_{12}$ della normale 

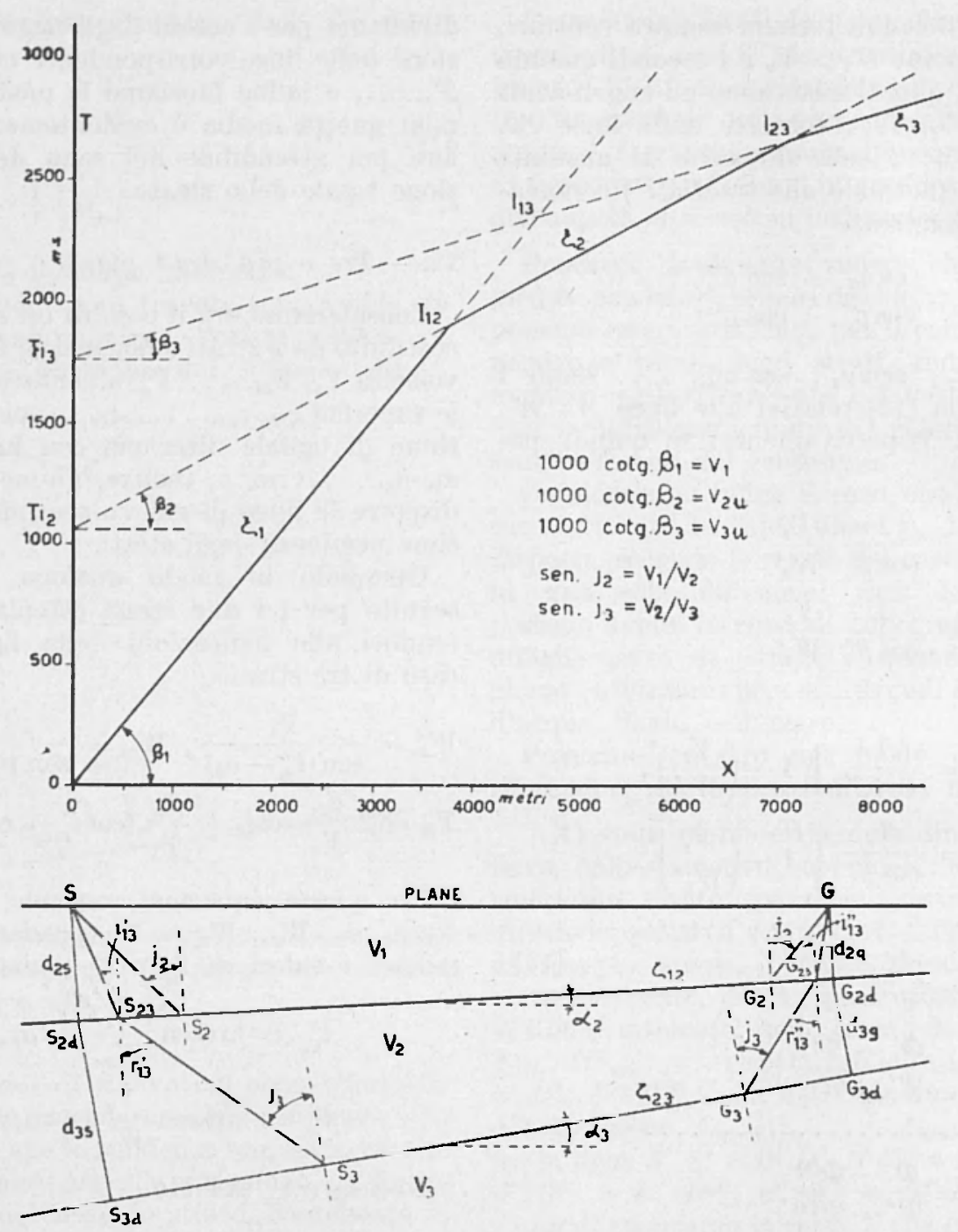

Fig. 7 - Raggi sismici e dromocrone di un sottosuolo costituito da tre strati piani nella direzione della massima pendenza.

alla $\varsigma_{12}$ stessa calata dal punto di scoppio $S$ ponendo

$$
d_{3 s}=\frac{\frac{V_{3}}{V_{2}}}{\sqrt{\left(\frac{V_{3}}{V_{2}}\right)^{2}-1}} V_{2} \frac{T_{i 3 r}}{2}
$$

ove

$$
T_{i 3 r}=T_{i 3}-\frac{d_{2 s}}{V_{1}}\left(\cos i_{13}^{\prime}+\cos i_{33}^{\prime \prime}\right)
$$

Pel caso di un suolo costituito da quattro strati abbiamo

$$
\begin{aligned}
& W_{4 u}=\frac{V_{1}}{\operatorname{sen}\left(i_{14}^{\prime \prime}-a_{2}\right)}, W_{4 d}=-\frac{V_{1}}{\operatorname{sen}\left(i_{14}^{\prime}-a_{2}\right)}, \\
& T_{i 4}=2 \frac{d_{4 s}}{i_{3}} \cos j_{4}+\frac{d_{2 s}}{V_{2}}\left(\cos i_{24}^{\prime}+\cos i_{24}^{\prime \prime}\right)+
\end{aligned}
$$$$
+\frac{d_{2 s}}{r_{1}}\left(\cos i_{14}^{\prime}+\cos i_{14}^{\prime \prime}\right) \text {. }
$$ 
Dalle prime due otteniamo

$$
\begin{aligned}
& i_{14}^{\prime \prime}=\operatorname{arcsen} \frac{V_{1}}{W_{-\prime}}+a_{2} \\
& i_{14}^{\prime}=\operatorname{arcsen} \frac{V_{1}}{W_{4 d}}-a_{2}
\end{aligned}
$$

$j_{1}+\left(\alpha_{1}-\alpha_{3}\right)=\operatorname{arcsen}\left[\frac{V_{3}}{V_{2}} \cdot \operatorname{sen}\left(r_{14}^{\prime}-a_{3}+a_{2}\right)\right]$ $j_{4}-\left(a_{1}-a_{3}\right)=\operatorname{arcsen}\left[\frac{V_{3}}{V_{2}} \operatorname{sen}\left(r_{14}^{\prime \prime}+a_{3}-a_{2}\right)\right]$, ove

$$
\begin{aligned}
& r_{14}^{\prime}=\operatorname{arcsen}\left(\frac{V_{2}}{V_{1}} \operatorname{sen} i_{1 /}^{\prime}\right) \\
& r_{14}^{\prime \prime}=\operatorname{arcsen}\left(\frac{V_{a}}{\overline{V_{1}}} \operatorname{sen} i_{1, \mid}^{\prime \prime}\right),
\end{aligned}
$$

e quindi

$$
\begin{aligned}
& \frac{V_{3}}{V_{1}}=\operatorname{sen} \frac{1}{2}\left\{\operatorname{arcsen}\left[\frac{V_{3}}{V_{2}} \operatorname{sen}\left(i_{12}^{\prime}-a_{3}+a_{2}\right)\right]+\right. \\
& \left.+\operatorname{arcsen}\left[\frac{V_{3}}{v_{2}} \operatorname{sen}\left(r_{14}^{\prime \prime}+\alpha_{3}-\alpha_{2}\right)\right]\right\} \\
& \alpha_{4}=a_{3}+\frac{1}{2}\left\{\operatorname{arcsen}\left[\frac{V_{3}}{V_{2}} \operatorname{sen}\left(r_{14}^{\prime}-a_{3}+a_{2}\right)\right]-\right. \\
& \left.-\operatorname{arcsen}\left[\frac{V_{3}}{V_{2}} \operatorname{sen}\left(r_{1:}^{\prime \prime}+\alpha_{3}-a_{2}\right)\right]\right\} \text {. }
\end{aligned}
$$

Per il calcolo della distanza $d_{4 s}$ otteniamo infine

$$
d_{y=}=\frac{\frac{V_{2}}{V_{3}}}{\sqrt{\left(\frac{V_{4}}{V_{3}}\right)^{2}-1}} V_{3} \frac{T_{i 4 r}}{2},
$$

ove

$$
\begin{aligned}
T_{i_{i j}}^{\prime}= & T_{i_{4}}-\frac{d_{2 s}^{25}}{V_{1}}\left(\cos i_{14}^{\prime}+\cos i_{14}^{\prime \prime}\right)- \\
- & \frac{d_{3 s}}{V_{2}}\left\{\operatorname { c o s } \left[r_{14}^{\prime}-\left(a_{3}-a_{2}\right) \mid+\right.\right. \\
& \left.\left.+\cos \mid r_{14}^{\prime \prime}+\left(a_{3}-a_{2}\right)\right]\right\} .
\end{aligned}
$$

Operando in modo analogo possiamo facilmente ricavare le formole relative a cinque strati e a un numero qualsiasi di strati.

Per gli effetti pratici dobbiamo osservare che il procedimento di cui sopra è da considernrsi valido soltanto nel caso che gli strati del sottosuolo abbiano tutti la stessa direzione e che le linee di rilievo siano disposte secondo la massima pendenza: caso che in pratica sicuramente si presenta assai di rado e che, d'altronde, non è dato di riscontrare in alcun modo direttamente dai dati di rilievo.

Quando gli strati avessero la stessa pendenza, e quindi avessero le superfici limiti parallele, il procedimento è invece valido qualunque sia l'orientamento delle linee di rilievo, naturalmente riferendo i dati delle pendenze e delle profondità ai piani normali agli strati contenenti le tracce delle linee di rilievo stesse sul P. B. In tali condizioni risulta, evidentemente,

$$
r_{13}^{\prime}=r_{13}^{\prime \prime}, r_{14}^{\prime}=r_{14}^{\prime \prime}, \ldots
$$

e quindi

$$
\begin{aligned}
& \frac{V_{a}}{V_{3}}=\frac{V_{9}}{V_{1}^{-}} \operatorname{sen} i_{13}^{\prime}=\frac{V_{2}}{V_{1}} \operatorname{sen} i_{13}^{\prime \prime} \\
& \frac{V_{3}}{V_{1}}=\frac{V_{3}}{V_{1}^{\prime}} \operatorname{sen} i_{14}^{\prime}=\frac{V_{3}}{V_{1}} \operatorname{sen} i_{14}^{\prime}
\end{aligned}
$$

Per le distanze otteniamo i tempi intercetti ridotti

$$
T_{i 3 r}=T_{t 3}-2 \frac{d_{2 s}}{T_{1}^{r}} \cos i_{13}^{\prime}
$$

$$
T_{i 4 r}=T_{i 1}-2 \frac{d_{2 s}}{V_{1}} \cos i_{14}^{\prime}-2 \frac{d_{3 s}}{V_{2}} \cos r_{14}^{\prime}
$$

Un'idea dell'approssimazione secondo la quale è verificata l'ipotesi del parallelismo degli strati si ha dagli scarti con cui risultano soddisfatte le [23].

I dati delle pendenze e delle distanze relativi a diverse linee sono componibili, nel caso degli strati paralleli, secondo le stesse regole di cui abbiamo detto al paragrafo precedente.

Nel caso del suolo costituito da tre strati orizzontali, per cui $\alpha_{2}=\alpha_{3}=0$, abbiamo

$$
\begin{gathered}
W_{2_{u}}=W_{2 d}=V_{2}, W_{3 u}=W_{3 d}=V_{3} \\
T_{i 2}=2 \frac{d_{2 s}}{V_{1}} \sqrt{1-\left(\frac{V_{1}}{V_{2}}\right)^{2}} \\
T_{i 3}=2 \frac{d_{3 s}}{V_{2}} \sqrt{1-\left(\frac{V_{2}}{V_{3}}\right)^{2}+2 \frac{d_{2 s}}{V_{1}} \sqrt{1-\left(\frac{V_{1}}{V_{3}}\right)^{2} .}}
\end{gathered}
$$


Le dromocrone dei tre strati hanno evidentemente le equazioni

$T=\frac{X}{V_{1}}, T=T_{i 2}+\frac{X}{V_{2}}, T-T_{i 3}+\frac{X}{V_{3}}$.

La prima dromocrona interseca la seconda e la terza rispettivamente nei punti $I_{12} \mathrm{e}$ $I_{13}$ di coordinate

$$
\begin{gathered}
X_{12}=2 d_{2 s} \frac{\sqrt{1-\left(\frac{V_{1}}{V_{2}}\right)^{2}}}{1-\frac{V_{1}}{V_{2}}}, T_{12}=\frac{X_{12}}{V_{1}}, \\
X_{13}=2 d_{y s} \frac{\sqrt{1-\left(\frac{V_{1}}{V_{3}}\right)^{2}}}{1-\frac{V_{1}}{V_{3}}}+2 d_{3 s} \frac{\sqrt{1-\left(\frac{V_{2}}{V_{3}}\right)^{2}}}{\frac{V_{2}}{V_{1}}-\frac{V_{2}}{V_{3}}} \\
T_{13}=\frac{V_{13}}{V_{1}} .
\end{gathered}
$$

Risulta di conseguenza la condizione per la quale $\mathrm{i}$ valori $\mathrm{di} X_{13}$ sono minori di quelli di $X_{12}$

$$
\begin{gathered}
\frac{d_{3 s}}{d_{2 s}}<\left\lfloor\frac{\frac{V_{3}}{V_{1}}-1}{\frac{V_{2}}{V_{1}-1}} \sqrt{\left(\frac{V_{2}}{V_{1}}\right)^{2}-1}-\right. \\
\left.-\sqrt{\left(\frac{V_{3}}{V_{1}}\right)^{2}-1}\right\rfloor /\left(\frac{\frac{V_{3}}{V_{1}}}{V_{2}}\right)^{2}-1 .
\end{gathered}
$$

Nella fig. 8 riportiamo la riduzione fotografica del grafico-prontuario di calcolo dei valori per i quali i due punti d'intersezione risultano coincidenti, ossia $X_{12}=X_{13}$, costruiti in base all'equazione che risulta dalla [26] ponendovi uguali i due membri.

Quando la [26] è verificata risulta evidentemente che l'energia trasmessa dal secondo strato lungo il contatto col primo strato non può essere rilevata che come secondo impulso, dopo quella trasmessa direttamente dal primo strato, per le minori distanze, e dopo quella trasmessa dal terzo strato lungo il contatto col secondo strato, per le maggiori distanze. Come si sa, i secondi impulsi solo raramente riescono di ampiezze sufficienti per un sicuro rilievo dai sismogrammi, specialmente quando appaiono prossimi a quelli delle onde dirette.

Mancando i dati relativi agli impulsi trasmessi dal secondo stirato la presenza di questo evidentemente sfuggirà nella ricostruzione tettonica e si sarà indotti a innalzare notevolmente il terzo strato in modo che, considerando i terreni soprastanti tutti pertinenti al primo strato di velocità minore di quella effettiva del secondo strato, risultino ugualmente soddisfatti i tempi osservati.

Per ognuno degli strati del sottosuolo possiamo evidentemente fare delle considerazioni analoghe: dei dati approssimativi possono essere ricavati dalla [26] sostituendo a $V_{1}$ la velocità dello strato immediatamente soprastante e a $d_{2 s}$ lo spessore totale degli strati soprastanti.

Le stesse formole possono considerarsi valide anche pel caso degli strati leggermente inclinati sostituendo alle velocità vere le velocità apparenti corrispondenti.

Per rimediare alle lacune che in conseguenza risultano per i rilievi di prospezione si cercherà di iniziare $\mathrm{i}$ rilievi delle dromocrone nelle zone per le quali i tempi delle stesse appaiono come primi impulsi ed estendere gradualmente $\mathrm{i}$ rilievi nelle zone per le quali i tempi appaiono come secondi impulsi in modo che questi trovino conferma negli allineamenti delle prime dromocrone.

Per gli effetti pratici è importante notare che se in una zona si riscontra la presenza, in qualche parte, ove le condizioni sono favorevoli, di un dato orizzonte rifrangente questo si deve in generale supporre presente in tutta la zona quando dai dati di rilievo si possono ritenere operanti le condizioni postulate colla [26]. Le dromocrone relative a questi orizzonti di regola avranno velocità inferiori a quelle degli impulsi precedenti.

\section{3. - Faglie degli strati piani.}

Come abbiamo visto al par. 9, ogni soluzione della continuità dei mezzi di trasmissione delle onde dà luogo ai fenomeni della diffrazione. Ora vediamo in dettaglio come tali fenomeni si esplicano nel caso che 
degli strati piani con velocità costante siano interrotti da una faglia.

Il sottosuolo sia costituito da due soli strati e la faglia, verticale di rigetto $\varrho$, non dia luogo a nessuna discontinuità dei mezzi di trasmissione quando, lungo la superficie di faglia $\sigma$, sono a contatto terreni della medesima specie. assottigliamento del primo strato quali risultano rispettivamente per le stazioni di scoppio $S^{\prime}$ ed $S^{\prime \prime}$ della fig. 9 che riportiamo nella pagina che $\cdot$ segue.

Nel caso degli scoppi in $S^{\prime}$ dì tale figura, le onde rifratte nel secondo strato raggiungono i diversi punti della faglia $F^{\prime} F^{\prime \prime}$, seguendo i raggi trasmessi secondo le nor-

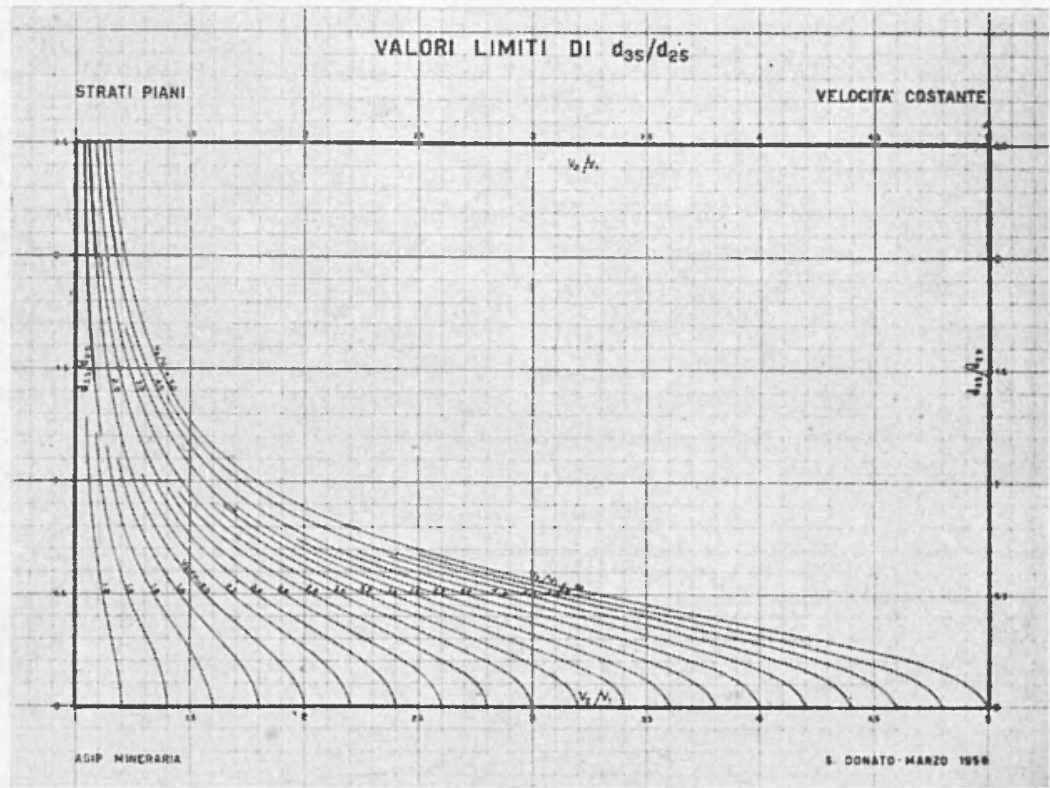

Fig. 8 - Prontuario di calcolo delle condizioni limiti di rilievo del secondo strato con primi oppure secondi impulsi.

In tali condizioni evidentemente le onde dirette, che si trasmettono nel primo strato, si propagano secondo impulsi continui, con intensità variabili regolarmente in conformità del normale decremento sferico e dell'assorbimento specifico del mezzo costituente il primo strato.

Le onde rifratte nel secondo strato, le quali evidentemente si trasmettono colle stesse modalità di cui abbiamo detto al par. 11 fino a tanto che non raggiungono la faglia, in corrispondenza alla faglia stessa danno luogo a deformazioni diverse delle dromocrone a seconda del senso dello spostamento dei due lembi; per chiarezza noi considereremo quindi separatamente i due rasi: che le onde incontrino in corrispondenza alla faglia un ispessimento oppure un mali leggi della rifrazione, con ampiezze rapidamente crescenti da $F^{\prime}$ verso $F^{\prime \prime}$.

Ora, l'energia irrradiata da F', di per sè molto debole, diminuirà approssimativamente in ragione della distanza e quindi arriverà ai sismografi posti dopo il punto $A$ con piccole ampiezze. $\mathrm{Al}$ confronto molto grandi saranno invece, per la [t], le ampiezze delle onde provenienti dalla superficie adiacente di traccia $F^{\prime} F^{\prime \prime}$.

La dromocrona delle onde rifratte dal secondo strato, rettilinea fino al punto $A^{\prime}$ corrispondente di $A$, subirà, a partire dallo stesso punto $A^{\prime}$, una inflessione graduale verso l'alto, tendendo asintoticamente a disporsi parallela a quella delle onde dirette: nella fig. 9 abbiamo indicato con $\xi^{\prime}$, tale dromocrona. Le ampiezze corrispondenti 
alla stessa dromocrona saranno in generale funzioni crescenti delle lugnhezze d'onda considerate e del rigetto $\varrho=F^{\prime} F^{\prime \prime}$ della faglia.
La distanza fra queste due dromocrone misurata in senso verticale (parallelo all'asse dei tempi), supposta trascurabile la differenza $\delta^{\prime} T$ fra i tempi che i raggi im-

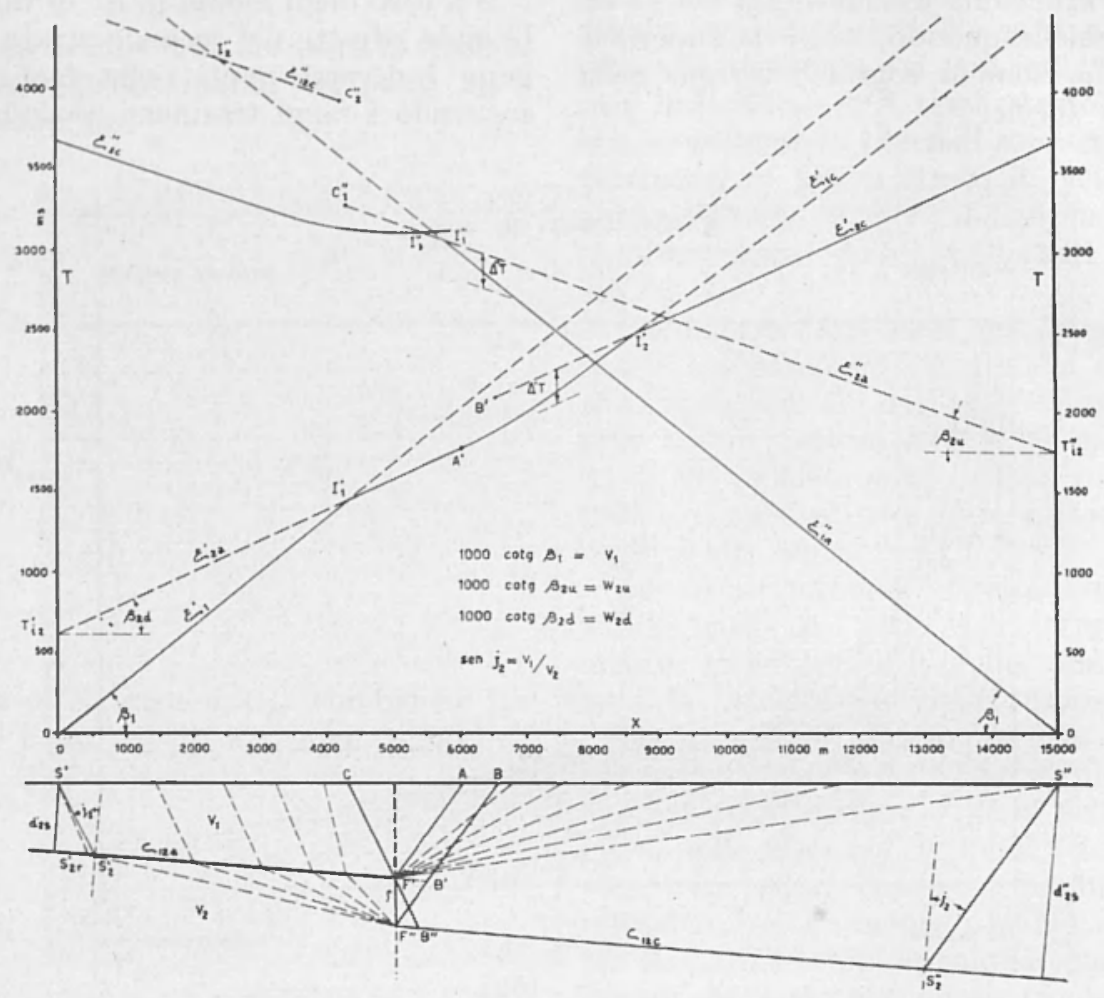

Fig. 9 - Raggi sismici e dromocrone di una faglia $F^{\prime} F^{\prime \prime}$ di un sottosuolo costituito da due strati piani.

La superficie di contatto $\varsigma_{12 c}$ fra il primo e il secondo strato che si trova dopo la faglia è tutta in "ombra ", stando all'ottica geometrica, e quindi non dovrebbe dar luogo a nessun impulso alla superficie del suolo. Praticamente invece, per effetto della solita diffrazione, avremo dell'energia sia per irraggiamento del punto $F^{\prime \prime}$ al sopraggiungere dell'impulso trasmesso lungo il contatto dei due strati sia in conseguenza dell'incurvamento dei raggi rifratti nel secondo strato verso la superficie $\zeta_{12 c}$. La dromocrona che risulterà, $\xi_{2 c}$, sarà evidentemente parallela a quella $\xi_{2 a}^{\prime}$ relativa al tratto del secondo strato che si trova prima della faglia. Le ampiezze saranno ancora funzioni crescenti delle lunghezze delle onde considerate. piegano a percorrere i tratti $S^{\prime} S^{\prime}{ }_{0} F^{\prime}$ ed $S^{\prime} S^{\prime}{ }_{27} F^{\prime \prime}$,

$\delta^{\prime} T=\left(\frac{S^{\prime} S_{2 r}^{\prime}}{V_{1}}+\frac{S_{2 r}^{\prime} F^{\prime \prime}}{V_{2}}\right) \quad\left(\frac{S^{\prime} S_{2}^{\prime}}{V_{1}}+\frac{S_{2}^{\prime} F^{\prime}}{V_{2}}\right)$

può essere calcolata ponendo

$$
\left.\Delta^{\prime} T=\frac{\varrho \cos \alpha_{2}}{V_{1}}\right) \longdiv { 1 - ( \frac { V } { V _ { 2 } } ) ^ { 2 } } - \frac { \varrho \operatorname { s e n } \alpha _ { 2 } } { V _ { 2 } } \text {. }
$$

Viceversa, noto dai dati di rilievo il valore della distanza $\Delta^{\prime} T$ fra le dromocrone $\xi_{2 a}^{\prime}$ e $\xi_{2 c}^{\prime}$, possiamo determinare il rigetto della faglia ponendo

$$
\varrho=\frac{V_{2} \Delta^{\prime} T}{\cos \alpha_{2} /\left(\frac{V_{2}}{V_{1}}\right)^{2}-1-\operatorname{sen} \alpha_{0}}
$$


Nel caso dello scoppio in $S^{\prime \prime}$ della fig. 9, stando all'ottica geometrica, dovrebbero risultare: a) l'impulso trasmesso dalle onde dirette, della dromocrona $\left.\xi_{1 n}^{\prime \prime}, b\right)$ quello delle onde trasmesse dal secondo strato al contatto col primo nel tratto che precede la faglia, della dromocrona $\left.\xi_{2 a}^{\prime \prime}, c\right)$ quello che risulta dalle onde dirette che si irradiano nel secondo strato attraverso la superficie di faglia lungo $F^{\prime} F^{\prime \prime}$, della dromocrona $\xi_{12 c}^{\prime \prime}$. A questi impulsi, per effetto della diffrazione, si somma quello della dromocrona $\xi_{2 c}^{\prime \prime}$ che risulta dalle onde irradiate da $F^{\prime \prime}$, al sopraggiungere dell'impulso della dromocrona $\xi_{2 a}^{\prime \prime}$, e dai raggi del secondo strato incurvati verso l'alto di cui già abbiamo detto a proposito delle dromocrone $\xi_{1 c}^{\prime}$ e $\xi^{\prime}{ }_{2 c}$.

La dromocrona $\xi^{\prime \prime}{ }_{2 c}$ tende a disporsi asintoticamente parallela alla $\xi_{2 a}^{\prime \prime}$ : la distanza $\Delta^{\prime \prime} T$ che risulta fra la $\xi^{\prime \prime}{ }_{2 a}$ e l'asintoto di $\xi^{\prime \prime}{ }_{2 c}$ si ottiene ponendo

$$
\Delta^{\prime \prime} T=\frac{\varrho \cos \alpha_{2}}{V_{1}} \sqrt{1-\left(\frac{V_{1}}{V_{2}}\right)^{2}}+\frac{\varrho \operatorname{sen} a_{2}}{V_{2}} .
$$

Da questa ricaviamo il rigetto $\varrho$ nota la distanza $\Delta^{\prime \prime} T$

$$
\varrho=\frac{V_{2} \Delta^{\prime \prime} T}{\cos \alpha_{2} /\left(\frac{V_{2}}{V_{1}}\right)^{2}-1+\operatorname{sen} \alpha_{2}}
$$

Noti $\Delta^{\prime} T$ e $\Delta^{\prime \prime} T$ possiamo determinare il rigetto ponendo

$$
\varrho=\frac{V_{2}\left(\Delta^{\prime} T+\Delta^{\prime \prime} T\right)}{2 \cos \alpha_{2} \sqrt{\left(\frac{V_{2}}{V_{1}}\right)^{2}-1} .}
$$

e possiamo determinare la pendenza della superficie di contatto dei due strati ponendo

$$
\operatorname{tg} \alpha_{2}=\frac{\frac{\Delta^{\prime \prime} T}{\Delta^{\prime} T}-1}{\frac{\Delta^{\prime \prime} T}{\Delta^{\prime} T}+1} / \sqrt{\left(\frac{\bar{V}_{2}}{V_{1}}\right)^{2}-1}
$$

Quest'ultima formola può servire per controllare i valori di $\Delta^{\prime} T$ e $\Delta^{\prime \prime} T$ quando è nota per altra via la pendenza.

Nel caso di una faglia avente il lembo ribassato dalla parte verso cui gli strati si innalzano, invece che dalla parte verso cui gli strati si abbassano, possiamo ripetere tutte le considerazioni sopra fatte e quindi valgono le stesse formole trovate: [28] e [29] per gli scoppi in $S^{\prime}$, [30] e [31] per gli scoppi in $S^{\prime \prime}$ e [32] e [33] per gli scoppi in $S^{\prime}$ ed $S^{\prime \prime}$.

Al solito per rendere agevole il lavoro di elaborazione dei dati di rilievo noi abbiamo predisposto i grafici-prontuario di calcolo dei valori del rigetto $\varrho$ in base alle [29] e [31]. Come si vede dalle fotografie clie riportiamo nelle figure 10 e 11 delle pagine che seguono, con tali grafici, costituiti ognuno da dodici quadri corrispondenti a diversi valori della pendenza $\operatorname{tg} a_{2}$ degli strati, possiamo determinare $i$ valori corrispondenti alle diverse pendenze soltanto per interpolazione fra i valori dei diversi quadri.

La precisione raggiungibile con tali grafici è certamente scarsa, date le grandi scale adottate, però non inferiore a quella clie in effetto riesce possibile con questo genere di calcoli.

Poichè il rigetto è lineare colle differenze dei tempi delle dromocrone i grafici di calcolo del rigetto stesso possono essere ampliati a volontà moltiplicando $\mathrm{i}$ valori segnati di $\varrho$ e di $\Delta^{\prime} T$ e $\Delta^{\prime \prime} T$ per un fattore qualsiasi: naturalmente la precisione risulterà ridotta in proporzione dello stesso fattore. Utilizzando questo principio si calcoleranno i valori di o compresi fra 0 e $100 \mathrm{~m}$, i quali non sono dati direttamente dai grafici.

Osserviamo che la [29] si trasforma nella [31] mutata di segno quando

$$
\frac{V_{1}}{V_{2}}>\cos \alpha_{2}
$$

e che verificandosi tale condizione noi potremo determinare $\mathrm{i}$ valori di $V_{2} \Delta^{\prime} T$ utilizzando il grafico relativo al caso degli strati che si elevano verso lo scoppio della fig. 10 e apponendo il segno negativo.

Con un sottosuolo costituito da tre o più strati piani si ripeterà per ogni dromocrona quanto abbiamo visto per quella del secondo strato e si potranno applicare le stesse formole sopra trovate per ognuno degli strati, sostituendo a $V_{2}$ il valore della velocità reale corrispondente. Naturalmente, trattandosi della medesima faglia in generale si 
potrà supporre il rigetto uguale per tutti gli strati e quindi, a seconda dei casi, mediare $\mathbf{i}$ valori relativi ai diversi strati per
Le considerazioni di cui sopra sono valide soltanto nelle ipotesi che si operi nei piani normali alle superfici di contatto degli strati

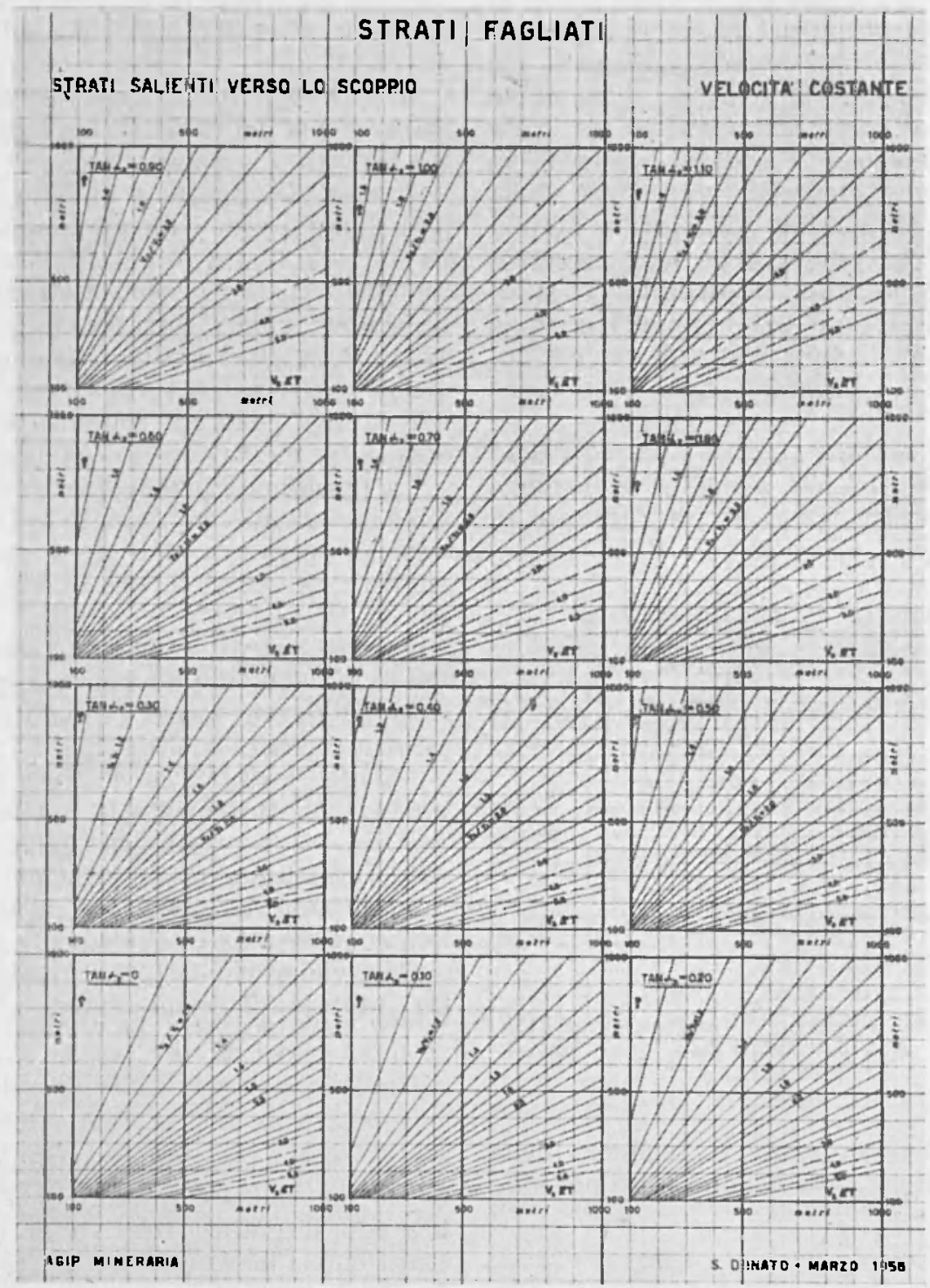

Fig. 10 - Prontuario di calcolo del rigetto $\varrho$ delle faglie nel caso dello scoppio dalla parte verso cui gli strati si elevano.

ottenere il valore più attendibile oppure utilizzare il valore che risulta di alcuni strati, per ricercare le dromocrone di altri da una parte e dall'altra delle faglie. e che le linee di rilievo siano perpendicolari alle faglie: ipotesi quest'ultima che raramente si trova verificata in pratica, almeno colla precisione necessaria. 
Con una linea di rilievo obliqua rispetto alla faglia avviene che i raggi rilevati dai sismografi sono corrispondenti a percorsi l'angolo di direzione $\delta$ della faglia stessa rispetto alla linea di rilievo.

Per una linea obliqua rispetto alla faglia

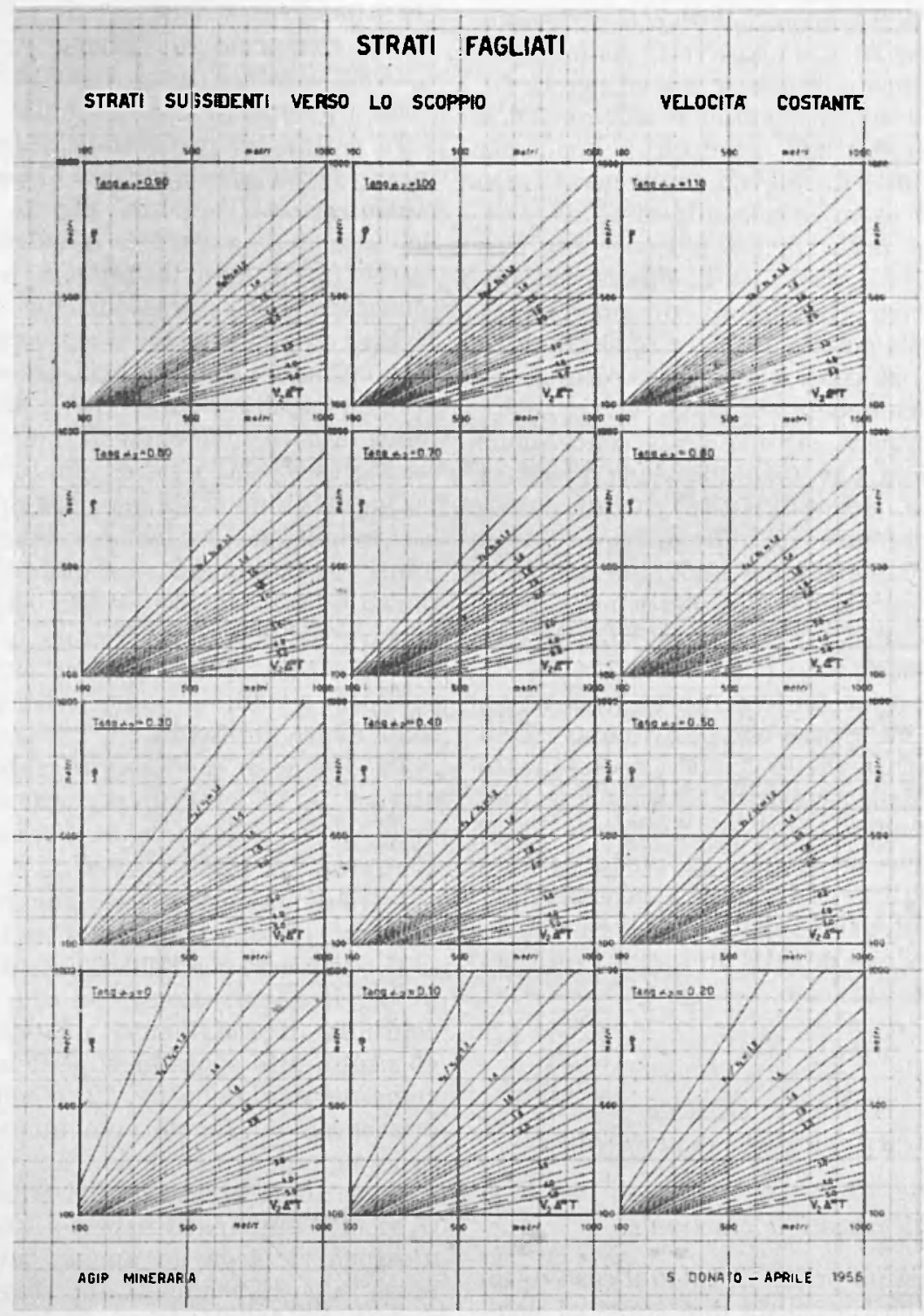

Fig. 11 - Prontuario di calcolo del rigetto $\varrho$ delle faglie nel caso dello scoppio dalla parte verso cui gli strati si abbassano.

affatto diversi, con nessun tratto in comune, i quali sono funzione oltrechè delle caratteristiche geometriche e litologiche degli strati e del rigetto della faglia anche del- risultano le seguenti caratteristiche peculiari:

1) angoli meno vivi fra le $\xi{ }_{9 n}$ e $\xi^{\prime \prime}{ }_{2 c}$ della fig. 9 . 
2) raccordi più ampi fra le $\varepsilon_{2 n}$ e $\xi^{\prime}{ }_{1 c}$,

3) incurvatura iniziale verso l'asse delle ascisse della $\xi_{2 c}^{\prime}$.

E da osservare però che le formole sopra trovate per il calcolo dei rigetti e i grafici delle figure 10 e 11 ricavati dalle stesse risultano ancora valide se anche per la $\xi_{2 c}^{\prime}$ della fig. 9 si considerano le distanze $\Delta^{\prime} T$ e $\Delta^{\prime \prime} T$ riferite agli asintoti, almeno per angoli fra linee di rilievo e faglie non troppo piccoli, per esempio non minori di $30^{\circ}$.

Vediamo dalla fig. 9 che per un solo strato fagliato risultano in effetto tre oppure quattro tronchi di dromocrone, a seconda della posizione dello scoppio rispetto alla faglia: se consideriamo che ogni strato presente comporta almeno altri tre o quattro tronchi ci rendiamo conto delle complicazioni che risultano dalla presenza delle faglie, delle difficoltà che di conseguenza sorgono nell'individuazione degli inizi degli impulsi sui sismogrammi e delle inevitabili incertezze che derivano nell'attribuzione degli stessi impulsi alle diverse dromocrone.

In pratica evidentemente si renderanno minime le incertezze che derivano ai rilievi dalla presenza delle faglie, specialmente agli effetti delle ricostruzioni tettoniche, rilevando le faglie stesse con numerose linee, possibilmente ortogonali, in modo da rendere possibili tutti i controlli necessari per la retta costruzione delle dromocrone e per l'individuazione di tutti gli impulsi utili per il dettaglio richiesto.

\section{STRATI CURVI CON VELOCITA COSTANTE}

\section{4. - Strati piegati a sinclinale.}

Consideriamo prima di tutto il caso di un sottosuolo costituito da due strati di velocità costante $V_{1}$ e $V_{2}$ a contatto secondo una superficie $\varsigma_{12}$ composta da due semipiani, normali al piano verticale per la linea di rilievo, di inclinazioni $90^{\circ}-\theta_{1}$ e $\theta_{2}-90^{\circ}$, raccordati secondo una superficie cilindrica circolare coll'asse orizzontale di raggio $R_{c}$.

Nelle condizioni rappresentate dalla fig. 12 se scoppiamo in $s^{\prime}$ otteniamo, dopo la solita dromocrona delle onde dirette, la dromocrona delle onde rifratte nel secondo strato colle stesse caratteristiche di cui abbiamo detto al par. 11 fino alla distanza corrispondente al sismografo $G_{1}$ : dal punto $R_{1}$ di rifrazione del raggio registrato da $G_{1}$ tutti i punti di $\varsigma_{12}$ sono in ombra per l'ottica geometrica.

In realtà, lungo l'arco $R_{1} R_{2}$ avremo dei raggi rifratti convergenti nel punto $C_{r}$ della circonferenza passante per la traccia $C$ dell'asse della superficie cilindrica e per le tracce $R_{1}$ ed $R_{2}$ delle rette di raccordo coi semipiani colla prosecuzione del raggio $R_{1} G_{1}$. come effetto della convergenza risulterà un aumento di energia pressapoco proporzionale al rapporto fra le distanze dei punti di rifrazione $R$ da $C_{r}$ e le distanze dei sismografi $G$ dallo stesso punto $\mathrm{C}_{r}$.

Questo aumento in generale prevarrà sulla diminuzione conseguente al fatto che i punti dell'arco sono in ombra quando il punto $C_{r}$ sarà prossimo alla superficie del suolo e nel medesimo tempo la curvatura dell'arco sarà piccola, ciò che può avvenire soltanto quando i punti dello stesso arco sono molto profondi.

In ogni caso, per quanto abbiamo detto al par. 9, si avrà un'accentuazione delle onde lunghe sulle corte per tutti i sismografi compresi fra quelli $G_{1}$ e $G_{2}$.

La dromocrona delle onde rifratte nel secondo strato si prosegue oltre il sismografo $G_{1}$, ammesso che l'energia risulti sufficiente per le registrazioni, con una variazione continua di andamento quando il centro $C$ risulta alto sopra la superficie del suolo, come nel caso della fig. 12 , e con una retta orizzontale ad angolo vivo quando $C_{r}$ cade sulla superficie del suolo. $\mathrm{Nel}$ caso che $C_{r}$ cada sotto la superficie del suolo il punto $G_{2}$ risulta anticipato rispetto a $G_{1}$ e la dromocrona si piega ad angolo vivo verso il basso in corrispondenza allo stesso $G_{2}$.

Quando fossero date le dromocrone e volessimo determinare la superficie $\varsigma_{12}$ di contatto fra il primo e il secondo strato possiamo operare nel seguente modo, riferendoci alle indicazioni della fig. 12, supposte note le velocità $V_{1}$ e $V_{2}$ degli strati:

1) calcoliamo il valore di $d_{2 s}$ dal tempo intercetto mediante la [11]; 
2) determinati dalle dromocrone $\varepsilon_{9 d} \mathrm{e}$ $\xi_{2 u}$ i valori delle velocità apparenti $W_{2 t} \mathrm{e}$ $W_{2 u}$ calcoliamo le pendenze corrispondenti di $c_{1}$ mediante i grafici delle figure 4 e 5 , $\operatorname{tg}\left(90^{\circ}-\theta_{1}\right)$ e $\operatorname{tg}\left(\theta_{2}-90^{\circ}\right)$ rispettivamente;
5) dal punto $R_{1}$ d'incontro delle due rette tracciamo la normale alla prima;

6) dal sismografo $G_{2}$, corrispondente al punto $A_{2}$ di variazione dell'andamento della $\xi_{2 c}$, tracciamo la retta che forma colla nor-
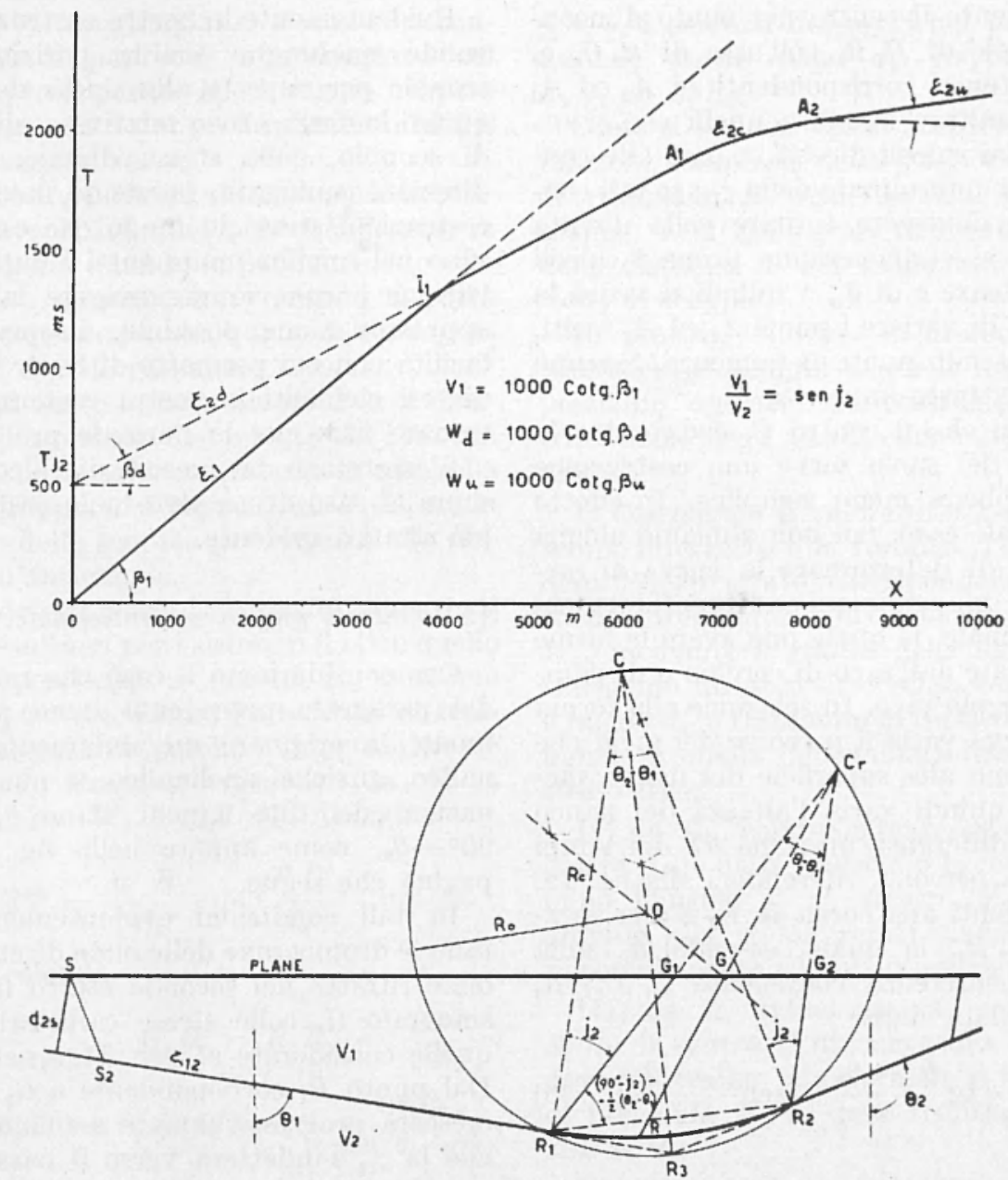

Fig. 12 - Raggi sismici e dromocrone di due strati piegati a sinclinale con piccola curvatura.

3) centro in $S$ tracciamo un arco di cerchio di raggio uguale al valore di $d_{2 s}$ determinato e tangente tracciamo la retta di pendenza $\operatorname{tg}\left(90^{\circ}-\theta_{1}\right)$;

4) dal sismografo $G_{1}$, corrispondente al punto $A_{1}$ di variazione dell'andamento della dromocrona $\xi_{2 d}$, tracciamo la retta che forma colla normale alla retta tracciata l'angolo $j_{2}-\operatorname{arcsen}\left(V_{1} / V_{2}\right)$; male alla retta $\varrho$ qualsiasi di pendenza tg $\left(\theta_{2}-90^{\circ}\right)$ l'angolo $j_{2}$, dalla parte delle $X$ crescenti: indichiamo con $C_{r}$ il punto d'incontro colla $R_{1} G_{1}$

7) tracciamo da $R_{1}$ la retta che forma l'angolo $90^{\circ}-j_{2}-\left(\theta_{2}-\theta_{1}\right) / 2$ colla $R_{1} C_{r}$ : sia $R_{2}$ il punto d'incontro colla $C_{r} G_{2}$;

8) tracciamo l'asse del segmento $R_{1} R_{2}$ : il punto d'incontro $O$ colla normale per $R_{1}$ 
alla retta di cui 3 ) è il centro del circolo di raccordo $R_{1} R_{2}$;

9) tracciamo tale arco e per $R_{2}$ tracciamo la retta di pendenza tg $\left(\theta_{2}-90^{\circ}\right)$;

10) come controllo i punti $R_{1}, R_{2}, C_{r}$ e $C$ devono risultare tutti su uno stesso circolo avente il centro nel punto d'incontro dell'asse di $R_{1} R_{2}$ coll'asse di $R_{1} C_{r}$ e, inoltre, i tempi corrispondenti ad $A_{1}$ ed $A_{2}$ devono risultare uguali a quelli che si ottengono dai calcoli diretti in base alle configurazioni determinate della $\varsigma_{12}$ : se tali controlli non dovessero tornare colla dovuta precisione si verificheranno prima i calcoli delle pendenze e di $d_{2 s}$ e quindi si vedrà la possibilità di variare i punti $A_{1}$ ed $A_{2}$ scelti, i quali, essendo punti di tangenza, saranno sempre piuttosto imprecisi.

Nel caso che il centro $C_{\text {r }}$ cada sotto la superficie del suolo serve una costruzione simile, sebbene meno semplice. In effetto però, in tale caso, noi non abbiamo alcuna possibilità di determinare la curva di raccordo fra i due semipiani costituenti i fianchi della sinclinale, la quale può avere le forme più disparate dall'arco di cerchio o di parabola all'angolo vivo. In relazione alla forma di tale curva varia il percorso dei raggi che si rifrangono alla superficie del fianco successivo e quindi varia l'altezza del fianco stesso: la differenza massima $\delta T$ dei tempi si ha per i percorsi, riferendoci alla fig. 12, corrispondenti alla corda $R_{1} R_{2}$ e alla spezzata $R_{1} R_{3} R_{2}$, la quale, essendo $R_{3}$ sulla stessa circonferenza contenente $C, C_{r}, R_{1}$ ed $R_{2}$, è data dalla

$$
\delta T=2 \frac{R_{c}}{V_{2}}\left(\operatorname{tg} \frac{\theta_{2}-\theta_{1}}{2}-\operatorname{sen} \frac{\theta_{2}-\theta_{1}}{2}\right) .
$$

Considerando, come noi abbiamo fatto, l'arco di cerchio $R_{1} R_{2}$ di centro $C$ di lunghezza circa media fra quelle della corda $R_{1} R_{2}$ e della spezzata $R_{1} R_{\mathrm{a}} R_{2}$ evidentemente avremo al massimo un errore metà.

Alzandosi il centro $C_{r}$ sopra la superficie del suolo, e quindi diminuendo la curvatura della piega diminuisce il valore di $\delta T$ e l'errore conseguente sulla profondità del piano opposto a quello dello scoppio; agli effetti pratici quindi noi possiamo ritenere che ammettendo la forma cilindrica circolare per i piegamenti non introduciamo delle cause di errori sensibili nei calcoli per la ricostruzione della superficie $\zeta_{12}$ quando le curvature dei piegamenti stessi e le pendenze dei fianchi non sono molto grandi, come avviene per la sinclinale della fig. 12.

Evidentemente le nostre costruzioni sono valide qualunque sia la posizione dello scoppio per rispetto alla sinclinale: avendo quindi le dromocrone relative a più stazioni di scoppio, nella stessa direzione e nelle direzioni coniugate, possiamo modificare le costruzioni stesse in modo che esse soddisfino nel miglior modo tutti i dati d'osservazione e con ciò raggiungere la migliore approssimazione possibile. Proprio per la facilità con cui permette di tener conto dei diversi elementi la nostra costruzione può tornare utile per la normale pratica.

L'estensione dei metodi di calcolo di cui sopra al caso di un sottosuolo costituito da più strati è evidente.

\section{5. - Strati piegati ad anticlinale.}

Ora consideriamo il caso che i due strati del paragrafo precedente siano piegati in modo da originare un andamento anticlinalico, anzichè sinclinalico, e che le inclinazioni dei due fianchi siano $\theta_{1}-90^{\circ} \mathrm{e}$ $90^{\circ}-\theta_{2}$, come appare nella fig. 13 della pagina che segue.

In tali condizioni evidentemente risultano le dromocrone delle onde dirette e delle onde rifratte nel secondo strato fino al sismografo $G_{1}$ colle stesse caratteristiche di quelle considerate al par. 11 e nella fig. 7 . Dal punto $R_{1}$ corrispondente a $G_{1}$ l'energia crescerà progressivamente a mano a mano che la $\varsigma_{12}$ s'infletterà verso il basso, fino a raggiungere il massimo in corrispondenza a un punto $R_{2}^{\prime}$, oltre $R_{2}$, per il quale risulta nullo l'effetto disperdente della superficie convessa della piega.

Per tracciare i raggi che si rifrangono dai punti compresi fra $R_{1}$ ed $R_{2}$ ai sismografi posti fra $G_{1}$ e $G_{2}$ possiamo utilizzare la nota costruzione di Weierstrass e quindi operare nel seguente modo:

1) concentrici al circolo $\gamma_{o}$, traccia della piega anticlinale, disegniamo $\mathrm{i}$ circoli $\gamma_{1}$ di raggio $R_{c} \cdot V_{1} / V_{2}$ e $\gamma_{2}$ di raggio $R_{c} \cdot V_{2} / V_{1}$ essendo $R_{c}$ il raggio di $\gamma_{o}$; 
2) congiungiamo il punto $R$ qualunque, compreso nell'arco $R_{1} R_{2}$, per il quale vogliamo tracciare il raggio rifratto $R G$, col punto $S_{2}^{\prime}$ di rifrazione nel secondo mezzo del raggio proveniente dallo scoppio, prolunghiamo fino a incontrare in $P$ il circolo $\gamma_{2}$, congiungiamo $P$ con $C$ e congiungiamo il punto $Q$ d'intersezione col circolo $\gamma_{1}$ col punto $R$ : il raggio rifratto cercato sarà dato dal prolungamento di $Q R$ fino alla superficie.

Il punto $S^{\prime}{ }_{2}$ varierà evidentemente in relazione alla posizione del punto $R$ fissato: in generale però, quando le pendenze e le curvature non saranno molto grandi, si potrà suppore coincidente con quello $S_{2}$ corrispondente ad $R_{1}$, di rifrazione totale.

Naturalmente le intersezioni $P$ e $Q$ risulteranno più o meno distanziate per i diversi punti $R$ in relazione alla curvatura della piega, al rapporto delle velocità $V_{1} / V_{0}$ e alla posizione dello scoppio per rispetto alla cerniera dell'anticlinale.

Per determinare le velocità apparenti $W_{d}$ che risultano per i sismografi oltre quello $G_{2}$ corrispondente al punto $R_{2}$ poniamo $d X=G_{2} G^{\prime}$, calcoliamo l'intervallo di tempo $d T$ necessario perchè l'impeto rifratto nel secondo strato si trasmetta da $G_{2}$ a $G^{\prime}$ e poniamo $W_{d}=d X / d T$. $\Lambda$ bbiamo

$$
d T=\frac{R^{\prime \prime} R^{\prime}}{V_{2}}+\frac{G^{\prime \prime} G^{\prime}}{V_{1}},
$$

per cui, essendo

$R_{2} R^{\prime}=\frac{\varrho_{2} d i}{\cos i}, G_{2} G^{\prime \prime}=R_{2} R^{\prime}+\frac{\varrho_{1} d r}{\cos r}$,

$R^{\prime \prime} R^{\prime}=R_{2} R^{\prime} \operatorname{sen} i, G^{\prime \prime} G^{\prime}=G_{2} G^{\prime \prime} \frac{\cos \theta_{2}}{\operatorname{sen}\left(\theta_{2}-r\right)}$, $d X=G_{2} G^{\prime \prime} \frac{\cos r}{\operatorname{sen}\left(\theta_{2}-r\right)}, d r=\frac{\operatorname{sen} r \cos i}{\operatorname{sen} i \cos r} d i$, risulta

$$
\frac{d X}{d i}-\frac{\varrho_{2} \operatorname{sen} i \cos ^{2} r+\varrho_{1} \operatorname{sen} r \cos ^{2} i}{\operatorname{sen} i \cos i \cos r \operatorname{sen}\left(\theta_{2}-r\right)}
$$

e di conseguenza

$$
W_{, l}=\frac{d X}{d i} \frac{d i}{d T}=\frac{V_{1}(1-f)}{\operatorname{sen}\left[\left(90^{\circ}-\theta_{2}\right)+r\right]-f \frac{\cos \theta_{2}}{\cos r},}
$$

essendo

$$
f=\frac{\varrho_{1}}{\varrho_{2}}-\frac{V_{1}}{V_{2}} \frac{1-\left(\frac{V_{2}}{V_{1}}\right)^{2} \operatorname{sen}^{2} r}{\cos ^{2} r},
$$

$\varrho_{1}$ e $\varrho_{2}$ i percorsi dei raggi nel primo e nel secondo strato dopo $S_{2}$, rispettivamente $R_{2} G_{2}$ ed $S_{2} R_{2}$.

Come si vede la velocità apparente $W_{d}$ è funzione complessa della posizione dei punti di rifrazione $R^{\prime}$ rispetto alla stazione di scoppio e ai sismografi riceventi e quindi delle distanze $X$ dei sismografi dalla stazione di scoppio.

In pratica, rilevate le dromocrone con caratteristiche simili a quelle della fig. 13, possiamo operare la ricostruzione geometrica della piega ad anticlinale nel seguente modo:

I) Calcoliamo il valore di $d_{2 s}$, mediante il tempo intercetto e la formola [11], e la pendenza dello strato corrispondente alla seconda dromocrona di velocità apparente $W_{2 u}$ mediante il grafico della fig. 4, quindi tracciamo un arco di cerchio con centro $S$ e raggio $d_{2 s}$ e tracciamo la retta di pendenza uguale a quella determinata tangente allo stesso arco;

II) da $G_{1}$, corrispondente al punto ${ }^{\circ}$ singolare $A_{1}$, e da $S$ tracciamo le rette che formino l'angolo $j_{2}-\operatorname{arcsen}\left(V_{1} / V_{2}\right)$ colla nornale alla stessa retta: siano $S_{2}$ ed $R_{1}$ rispettivamente i punti d'intersezione;

III) da $R_{1}$ tracciamo la normale alla $S . R_{1}$ : il centro $C$ di curvatura della piega deve cadere su tale normale e il circolo $\gamma_{1}$ di raggio $R_{c} \cdot V_{1} / V_{2}$ deve risultare tangente alla $G_{1} R_{1}$;

IV) determiniamo il centro $C$ per tentativi e ugualmente determiniamo il fianco discendente dell'anticlinale.

Quando avessimo anche le dromocrone coniugate, possiamo determinare direttamente entrambi i fianchi dell'anticlinale e il centro $C$ rimane automaticamente determinato: al solito converrà, naturalmente, adottare per $C$ un punto che concili coi minori scostamenti tutti i dati d'osservazione disponibili.

La costruzione di Weierstrass può essere applicata evidentemente qualunque sia il 
numero degli strati costituenti il sottosuolo.

Per il raso delle pieghe ad anticlinale è da osservare:

a) che le forme adottate per le pieghe non influenzano $i$ valori delle dromocrone
16. - Metodo degli angoli d'emergenza per l'elaborazione dei risultati.

La superficie $\varsigma_{12}$ della piega sinclinalica della fig. 12 puó essere sostituita da elementi di piani perpendicolari alla sezione
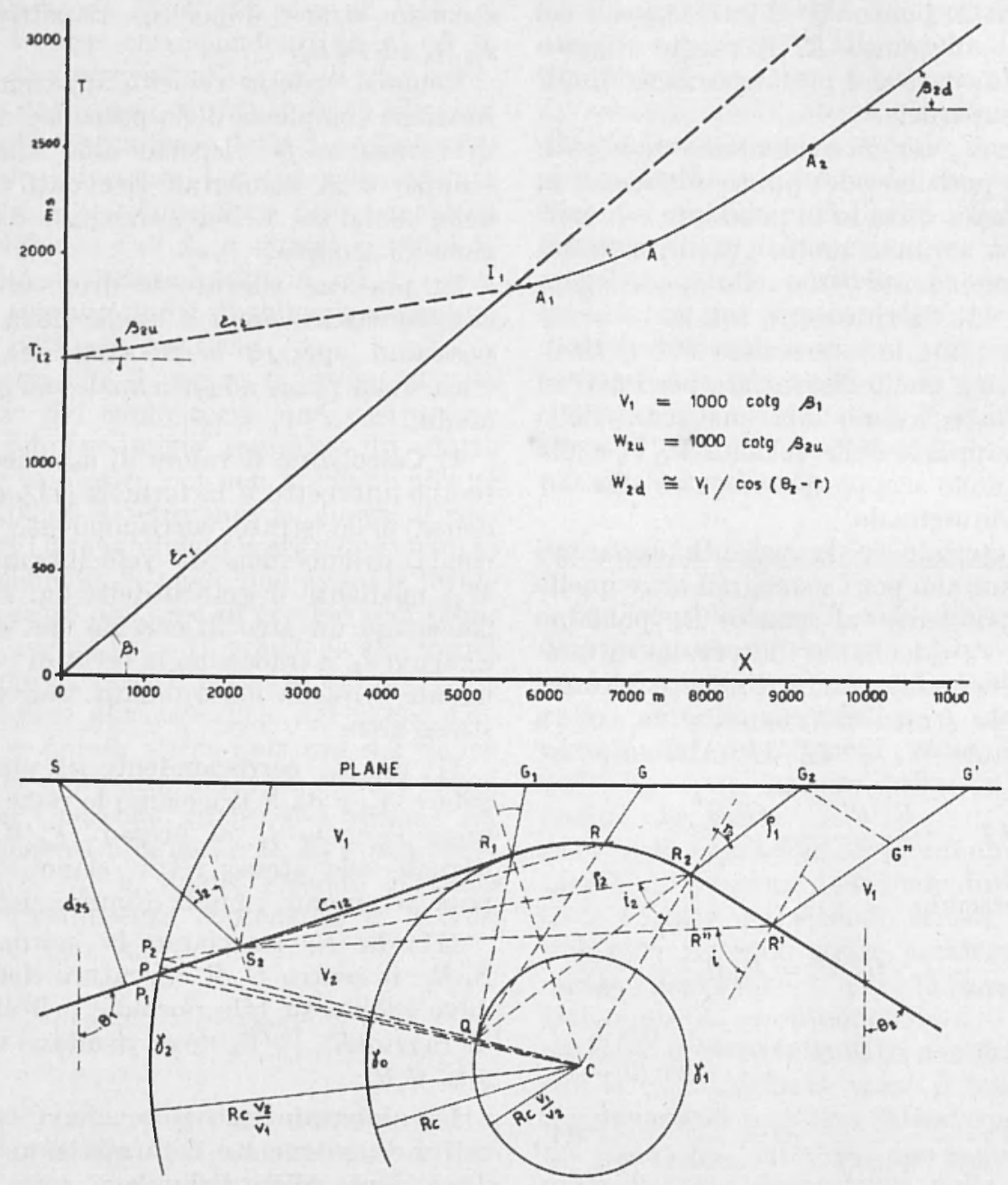

Fig. 13 - Raggi sismici e dromocrone di due strati piegati ad anticlinale.

e dei fianchi posti dalla parte opposta delle stazioni di scoppio;

b) i raggi rilevati dai diversi sismografi in corrispondenza alle cerniere e ai fianchi successivi non hanno nessun tratto in comune, sebbene siano corrispondenti alle medesime dromocrone. della figura i quali abbiano per tracce sulla stessa sezione i lati di una poligonale coi vertici sulla traccia della superficie $\varsigma_{12}$.

La dromocrona corrispondente a tale poligonale è un'altra poligonale avente i vertici sulla dromocrona $\xi_{2 u}$ corrispondente alla superficie $\varsigma_{12}$. 
Per ogni elemento di piano il tratto di dromocrona corrispondente ha evidentemente la velocità apparente che soddisfa le [8]. Poichè gli elementi di piano possono essere resi piccoli a piacere deduciamo che in effetto le velocità apparenti delle dromocrone e le inclinazioni della superficie $\varsigma_{12}$ soddisfano sempre le [8] qualunque sia la forma della stessa superficie.

In base a questo principio e alla possibilità di determinare, per le [8], [15], e [19], gli angoli di emergenza dei raggi dalle velocità apparenti gli autori hanno approntato il metodo generale di elaborazione dei risultati e di ricostruzione tettonica degli strati qui di seguito descritto, il quale per comodità può essere chiamato con " metodo degli angoli d'emergenza ".

Tale metodo, il quale presuppone note le velocità di trasmissione delle onde sismiche degli strati, consiste nelle seguenti operazioni, da eseguirsi nello stesso ordine con cui sono elencate:

1. - Rileviamo dai sismogrammi i diversi impulsi, riduciamo i valori osservati $T_{o}$ secondo i criteri specificati nei paragrafi 5 e 6 (utilizzando il modulo riportato al termine del par. 6, un foglio diverso per ogni impulso e per ogni scoppio, e tenendo conto di tutti gli accorgimenti suggeriti, in particolare di quelli relativi alla continuità delle sequenze e delle dromocrone corrispondenti) e quindi tracciamo le curve dromocrone di tutti gli impulsi rilevati in un unico diagramma colle ascisse alla scala 1:25.000 e le ordinate alla scala di $1 \mathrm{~cm}$ $=100 \mathrm{~ms}$;

2. - Consideriamo in primo luogo l'impulso relativo al secondo strato; quindi nei fogli che ne riportano i dati calcoliamo, nelle righe successive a quella dei tempi $T_{r}$ : le differenze $\Delta X$ fra i valori successivi delle distanze $X$ dei sismografi dalla stazione di scoppio, le differenze $\Delta T$ fra $i$ valori successivi dei tempi $T_{r}$ e i rapporti $W_{2}$ fra i valori di $\Delta X$ e $\Delta T$ delle medesime colonne, con tre cifre significative esatte (usando il regolo calcolatore): segniamo i valori a cavalcioni delle righe che separano le colonne ai quali sono relativi;

3. - Calcoliamo negli stessi fogli i valori di sen $\varphi_{2}$ corrispondenti alle velocità apparenti $W_{2}$ determinate ponendo

$$
\operatorname{sen} \varphi_{2}-\frac{T_{1}}{\eta_{2}}
$$

colla precisione della seconda cifra decimale esatta e calcoliamo i valori di $\operatorname{tg} \varphi_{2}$ corrispondenti;

4. - Infine calcoliamo i valori di $\operatorname{tg}_{m} \varphi_{2}$ medi fra quelli successivi di $\operatorname{tg} \varphi_{2}$, pure colla precisione della seconda decimale esatta, e li segniamo entro le colonne che risultano delimitate dalle coppie alle quali sono relativi: così operando evidentemente i valori $\operatorname{dit}_{n} \varphi_{2}$ delle diverse colonne risultano esattamente corrispondenti ai sismografi coi numeri che sono alle testate delle colonne stesse;

5. - Sulla sezione per i sismografi, disegnata sulla stessa carta delle dromocrone nel modo indicato dalla fig. 14, tracciamo a partire dai punti del P. B. corrispondenti ai sismografi $1,2, \ldots$ le semirette $\lambda_{1}, \lambda_{2}$, .... di pendenze, misurate rispetto alla verticale, uguali ai valori $\operatorname{di}_{\operatorname{tg}} \operatorname{tg}_{2}$ relativi alle medesime colonne e per i punti medi fra i sismografi 1 e 2,2 e $3, \ldots$ tracciamo le semirette $\mu_{1}, \mu_{2}, \ldots$ di pendenze uguali ai valori di $\operatorname{tg} \varphi_{2}$ che figurano nelle colonne relative ai sismografi 1 e 2,2 e $3, \ldots$;

6. - Per il punto $N_{2}$ di profondità nota, o supposta nota, tracciamo il segmento, delimitato dalle semirette $\mu_{n-1}$ e $\mu_{n}$ che comprendono $N_{2}$ stesso, il quale formi colla semiretta $\lambda_{n}$ per il sismografo compreso l'angolo $90^{\circ}-j_{2}$, essendo come sappiamo $j_{2}=$ arcosen $\left(V_{1} / V_{2}\right)$, coll'ausilio di una squadretta, appositamente costruita, in celluloide, cogli angoli acuti uguali a $j_{2}$ e $90^{\circ}-j_{2}$, e quindi dal punto $P_{n}$ d'incontro colla $\mu_{n}$ tracciamo, coll'ausilio della medesima squadretta, la semiretta che formi lo stesso angolo $90^{\circ}-j_{2}$ colla $\lambda_{n+1}$ fino a incontrare in $P_{n+1}$ la $\mu_{n+1}$ e così di seguito: la spezzata che in tale modo si ottiene non è altro che il profilo cercato della superficie $\varsigma_{12}$ di contatto fra il primo e il secondo strato, quando le condizioni di rilievo sono tali per cui risultano soddisfatte le [8];

7. - In effetto tali equazioni sono soddisfatte soltanto quando la superficie $\varsigma_{12}$ rivolge la concavità verso i sismografi di rilievo, o tutt'al più è piana, dato che solo per tali condizioni i raggi si trasmettono nel secondo strato lungo la superficie $\varsigma_{12}$ 
stessa; in pratica però si può sempre disporre le stazioni di scoppio in modo che le richieste condizioni siano verificate: per esempio, nella fig. 14 vediamo che ponendo lo scoppio alla stazione 3 risultano condizioni favorevoli per rilevare l'anticlinale sottostante mentre si hanno condizioni sfa- zone adiacenti; in ogni caso conviene calcolare il profilo coi dati di tutti gli scoppi possibili e adottare il profilo medio ponderale;

8. - Per la ricostruzione del contatto $\varsigma_{23}$ fra il secondo e il terzo strato, utilizzando naturalmente le dromocrone relative al-

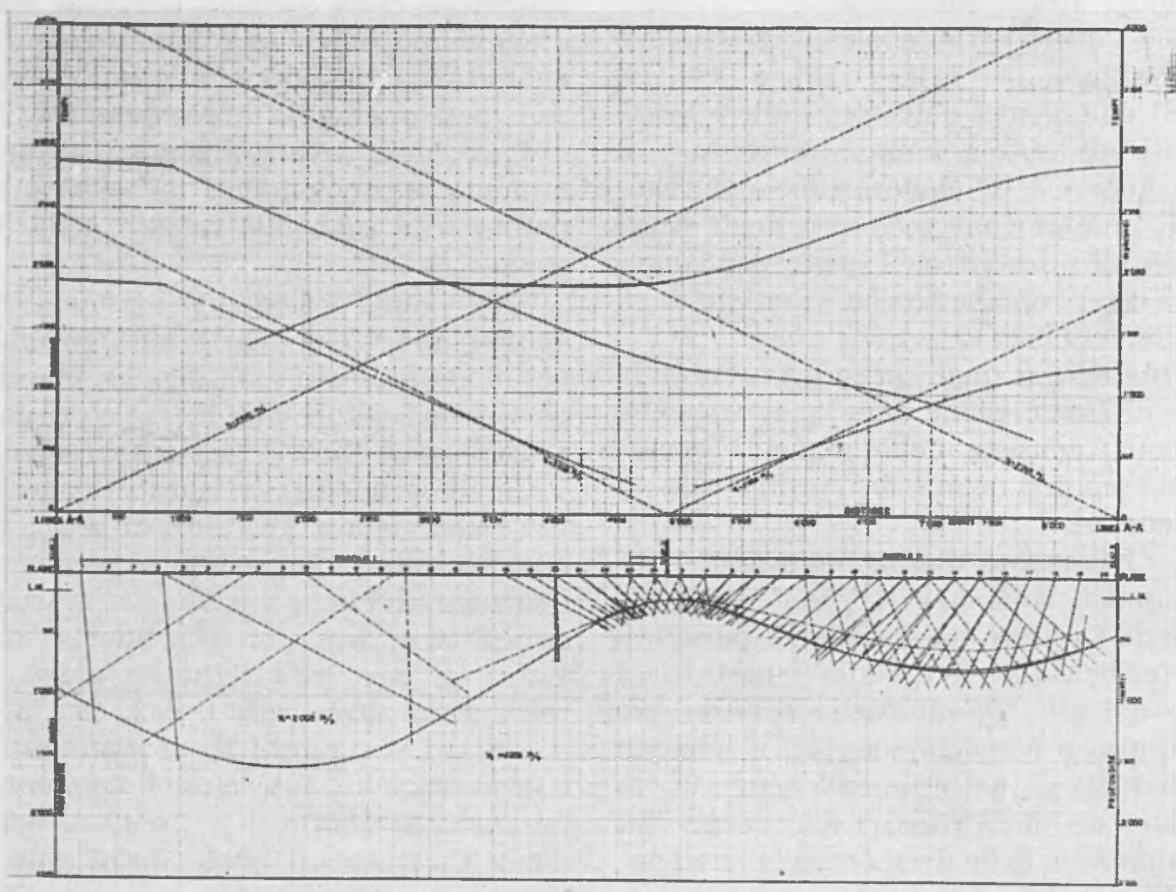

Fig. 14 - Confronto fra il profilo reale e quelli ricostruiti di una struttura anticlinalica.

vorevoli scoppiando alle stazioni 1 e 2 ; è da osservare che quando le [8] non risultano verificate i risultati della ricostruzione stessa lo dimostrano: vediamo infatti dalla fig. 14 che scoppiando nelle stazioni 1 e 2 si ottengono per i fianchi discendenti dell'anticlinale dei profili molto più elevati dei reali (segnati con linee tratteggiate) e che tuttavia fanno chiaramente vedere la concavità rivolta verso il basso; non disponendo di stazioni di scoppio in condizioni tali per cui risultino soddisfatte le [8] si può sempre, evidentemente, effettuare la ricostruzione calcolando direttamente il profilo in base ai tempi delle dromocrone e tenendo conto delle traiettorie dei raggi quali conseguono dalle configurazioni ammesse per le l'impulso corrispondente: A) riduciamo i valori osservati nello stesso modo indicato in 1 e costruiamo le dromocrone relative, B) calcoliamo le differenze $\Delta X$ delie distanze dai sismografi e le differenze $\Delta T$ dei tempi relativi e quindi calcoliamo le velocità apparenti $\left.W_{3}=\Delta X / \Delta T, \mathrm{C}\right)$ calcoliamo i valori $\mathrm{di}$

$$
\operatorname{sen} \varphi_{3}=\frac{V_{1}}{W_{3}}
$$

e quelli di $\operatorname{tg} \varphi_{3}$ corrispondenti,D) calcoliamo i valori di $\operatorname{tg}_{m} \varphi_{3}$ medi fra quelli successivi di $\left.\operatorname{tg} \varphi_{3}, \mathrm{E}\right)$ sulla sezione per i sismografi, ove sia segnato il contatto $\varsigma_{12}$ fra il primo e il secondo strato, tracciamo le semirette $\lambda_{1}$, $\lambda_{2}, \ldots, \mu_{1}, \ddot{\mu}_{2}, \ldots$ colle pendenze, misu- 
rate rispetto alla verticalo, uguali ai valori rispettivamente di $\operatorname{tg}_{m} \varphi_{3}$ e $\operatorname{tg} \varphi_{3}$ sopra calcolati esattamente come indicato in 5 per i valori relativi a $\varphi_{2}$, F) dai punti d'incontro $L_{1}, L_{2}, \ldots$ delle $\lambda_{1}, \lambda_{2}, \ldots$ colla $\zeta_{12}$ tracciamo le semirette $\lambda_{1}, \lambda_{2}, \ldots$ le quali formano gli angoli $r_{13}$ colle normali alla $\varsigma_{12}$ tali per cui

$$
\operatorname{sen} r_{13}=-\frac{V_{2}}{V_{1}} \operatorname{sen} i_{13},
$$

essendo $i_{13}$ gli angoli formati dalle $\lambda_{1}, \lambda_{2}, \ldots$ colle stesse normali alla $\zeta_{12}$, utilizzando per esempio il noto regolo di Shoeffer e Diemer di cui abbiamo detto al par. 8, e nello stesso modo tracciamo le semirette $\mu_{1}^{\prime}, \mu_{2}^{\prime}$, ... per i punti d'incontro $M_{1}, M_{2}, \ldots$. delle $\mu_{1}, \mu_{2}, \ldots$ colla $\left.\varsigma_{12}, \mathrm{G}\right)$ per il punto $N_{3}$ di profondità nota, o supposta nota, tracciamo il segmento delimitato dalle semirette $\mu_{n-1}^{\prime}$ e $\mu_{n}^{\prime}$ che comprendono $N_{3}$ stesso il quale formi colla $\lambda^{\prime}{ }_{n}$ l'angolo $90^{\circ}-j_{3}$, ove $j_{3}=\operatorname{arcosen}\left(V_{2} / V_{3}\right)$, coll'ausilio di una squadretta avente gli angoli acuti uguali a $j_{3}$ e $90^{\circ}-j_{3}$, e quindi dal punto $Q_{n}$ di incontro colla $\mu_{n}^{\prime}$ tracciamo, coll'ausilio della medesima squadretta, la semiretta che formi lo stesso angolo $90^{\circ}-j_{3}$ colla $\lambda^{\prime}{ }_{n+1}$ fino ad incontrare in $Q_{n+1}$ la $\mu_{n+1}^{\prime}$ e cosi di seguito: la spezzata che risulta è il profilo cercato della superficie $\varsigma_{23}, \mathrm{H}$ ) completiamo l'elaborazione del profilo della superficie $\varsigma_{23}$ tenendo conto di quanto abbiamo detto in $\tau$, valevole anche in questo caso;

9. - Per la ricostruzione del contatto $\varsigma_{34}$ fra il terzo e il quarto strato e per quelli successivi operiamo in modo analogo.

Per tracciare colle squadrette di celluloide speciali, di cui abbiamo detto in 6,8 e 9 , i segmenti della spezzata dei profili operiamo nel seguente semplice modo: 1) appoggiando un cateto della squadretta ad una riga comune facciamo coincidere l'altro cateto colla semiretta rispetto alla quale il segmento da tracciare deve formare l'angolo $90^{\circ}-j_{2}$, oppure $\left.90^{\circ}-j_{3}, 90^{\circ}-j_{4}, \ldots 2\right)$ giriamo la squadretta in modo che a contatto colla riga si trovi il cateto il quale forma l'angolo di $j_{2}$, oppure $j_{3}, j_{4}, \ldots$ colla ipotenusa, facciamo scorrere lungo la riga finchè il punto di partenza viene a cadere sull'ipotenusa e quindi tracciamo il segmento seguendo l'ipotenusa stessa.
Le semirette le quali formano le spezzate secondo cui i raggi si trasmettono ai sismografi di registrazione sul P.B. attraverso gli strati, saranno tracciate in modo corretto se si terrà presente: $a$ ) che gli angoli delle prime semirette, dipartentesi dai punti del P. B. devono essere misurati verso le stazioni di scoppio quando le velocità apparenti dello strato considerato sono positive e viceversa quando queste sono negative, b) che le spezzate in ogni caso devono volgere le "concavità" verso le stazioni di scoppio (almeno fino a tanto che si ammettono velocità per gli strati crescenti colle profondità degli strati stessi).

Per i tratti rettilinei delle dromocrone naturalmente si potrà, con vantaggio per la rapidità e la precisione, limitare i calcoli delle pendenze alle sole semirette per gli estremi degli stessi tratti e tracciare un segmento unico per il profilo del contatto degli strati.

Nella fig. 14 presentiamo la ricostruzione di una piega completa del contatto fra il primo e il secondo strato, effettuata col metodo di cui sopra, sui dati di quattro diverse dromocrone: due relative a una stazione di scoppio posta sulla cerniera dell'anticlinale e due relative a due stazioni di scoppio poste sulle sinclinali adiacenti. Gli elementi relativi alla stazione sulla cerniera, Staz. 3, vi sono segnati con linee sottili continue e quelli relativi alle stazioni sulle sinclinali, Staz. 1 e Staz. 2, con linee sottili tratteggiate.

Vediamo che le ricostruzioni della struttura nel tratto compreso fra le stazioni 3 e 2 effettuate in base alle dromocrone relative alle stesse stazioni portano a differenze massime, al fondo della sinclinale, di una trentina di metri, dell'ordine degli errori che ogni ricostruzione del genere comporta. Per il tratto compreso fra le stazioni 1 e 3 otteniamo, dalle dromocrone delle stesse stazioni, un profilo perfettamente concordante con quello dato per i fianchi della sinclinale mentre peril fondo della stessa sinclinale non riesce possibile nessuna ricostruzione, dato che i centri di curvatura risultano o più bassi del P. B. o di poco più alti dello stesso. In tali condizioni evidentemente le quote possono essere portate attraverso la sinclinale soltanto per interpolazione mediante 
il calcolo diretto dei tempi e dei percorsi, essendo i tempi relativi ai raggi rifratti dal fondo della sinclinale o mancanti o indistinti nelle dromocrone.

II profilo ricavato dalla dromocrona della Staz. 1 per il tratto compreso fra le stazioni 3 e 2, per le ragioni di cui abbiamo detto al par. 10, risulta molto più alto del reale però simile a quest'ultimo dal fondo della sinclinale in poi; l'asse dell'anticlinale risulta leggermente spostato verso la Staz. 2. Caratteristiche analoghe naturalmente si hanno per il profilo ricostruito in base alla dromocrona della Staz. 2 pel tratto fra le stazioni 3 ed 1 coll'aggiunta dell'interruzione in corrispondenza al fondo della sinclinale dovuta alla forte curvatura.

Le quote di partenza delle ricostruzioni potranno essere date dai sondaggi meccanici d'esplorazione eseguiti nella zona oppure da altri rillevi geofisici eseguiti, per esempio dai rllievi sismici a riflessione; di solito però avverrà che tali quote dovranno essere determinate mediante gli stessi rilievi a rifrazione, ciò che, per quanto sappiamo, si potrà fare con buona precisione se si disporrà di tutti i dati necessari, si conosceranno le velocità di trasmissione dei terreni colla dovuta precisione e si potrà escludere la presenza di faglie $o$ di altre interruzioni degli strati.

La forma dei profili varierà in generale colla quota di partenza scelta, data la diversa convergenza delle semirette per i sismografi di cui abbiamo detto in 5 e quindi variando le quote di partenza si dovranno rideterminare $\mathrm{i}$ profili tracciando le spezzate per $\mathrm{i}$ nuovi punti di partenza coi lati paralleli a quelli delle spezzate costituenti i primi profili.

Per le zone di tettonica movimentata, specialmente se ad elementi curvilenei, conviene adottare la scala di 1:10.000 per i disegni delle elaborazioni.

Le linee molto lunghe in zone di tettonica complessa, con andamenti sinclinalici e anticlinalici, come sappiamo non permettono la ricostruzione della tettonica stessa colla necessaria approssimazione: però in generale esse riescono ugualmente molto utili per dare un quadro d'insieme, e per disporre i programmi di rilievo più appropriati: agli efletti pratici quindi noi riteniamo che con- venga applicare il nostro metodo anche per tali linee, salvo naturalmente rifare la ricostruzione coi metodi più appropriati in sede di elaborazione finale dei rilievi.

\section{7. - Metodo di Gardner.}

Se nella [7] poniamo $a_{2}=0$ otteniamo, tenendo presente che sen $j_{2}=V_{1} / V_{2}$,

$$
T-\frac{X}{V_{2}}=T_{i 2}
$$

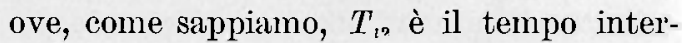
cetto.

L'espressione del tempo intercetto, quale risulta dalla stessa [7], può essere però posta sotto la forma

$$
T_{i 2}=2\left(\frac{1}{V_{1}} \frac{h_{2 s}}{\cos j_{2}}-\frac{1}{V_{2}} h_{2 s} \operatorname{tg} j_{2}\right),
$$

dalla quale vediamo che il tempo intercetto stesso non è altro che la differenza fra i tempi che i raggi impiegano a percorrere $i$ tratti entro il primo strato e quello che impiegherebbero a percorrere entro il secondo strato i tratti individuati dai punti di rifrazione e dalle proiezioni verticali dello scoppio e dei sismografi di registrazione.

Vediamo pure dalla [41] che in effetto il valore del tempo intercetto non varia se ammettiamo delle profondità diverse del secondo strato in corrispondenza allo scoppio e ai sismografi purchè sia costante la somma: possiamo quindi porre anche:

$$
\begin{aligned}
T_{i,} & =\left(\frac{1}{V_{1}} \frac{\lambda_{2 s}}{\operatorname{sen} j_{2}}-\frac{\lambda_{s s}}{V_{2}}\right)+ \\
& +\left(\frac{1}{V_{1}} \frac{\lambda_{2 g}}{\operatorname{sen} j_{2}}-\frac{\lambda_{2 g}}{V_{\eta}}\right),
\end{aligned}
$$

essendo indicati con $\lambda_{2 s}$ e $\dot{\lambda}_{2 g}$ gli offsets dello scoppio e dei sismografi, ossia, rispettivamente, le distanze orizzontali fra la proiezione verticale dello scoppio sul secondo strato e il punto di rifrazione totale dei raggi e fra i punti di rifrazione dei raggi ai sismografi e le proiezioni verticali degli stessi sul secondo strato.

Chiameremo anche noi, come l'autore del metodo (Gardner 1939), con delay time dello scoppio e dei sismografi rispettivamente le coppie di termini racchiuse nelle 
parentesi che si riferiscono allo scoppio e ai sismografi.

Con strati leggermente inclinati, per esempio con inclinazioni minori di $10^{\circ}$, la [40] è ancora verificata, almeno entro i limiti dell'approssimazione che in pratica possiamo ammettere, e, d'altra parte, le variazioni che conseguono negli offsets, ossia nelle posizioni dei punti di rifrazione dei raggi, comportano delle variazioni nei valori dei delay times minime, del secondo ordine, talchè si può ammettere che pure la [42] sia ancora verificata con sufficiente approssimazione.

Risulta evidente allora come si possa in pratica determinare la quota del contatto fra il primo e il secondo strato quando i piegamenti dello stesso sono a piccole pendenze ed è nota la quota in corrispondenza alla stazione di scoppio. In pratica possiamo operare nel modo seguente:

I) Rilevate le dromocrone colle stesse modalità indicate per il metodo degli angoli d'emergenza calcoliamo, su un modulo apposito, relativo all'impulso del secondo strato, i rapporti fra le distanze $X$ segnate nella prima riga (non ridotte di $\delta X$ ) colla velocità $V_{2}$ dello stesso secondo strato e alla riga successiva segniamo le differenze fra i valori ottenuti e i tempi ridotti $T_{r}$ : in tale modo abbiamo i valori del tempo intercetto $T_{\imath}$, secondo la [40] relativi ai diversi sismografi;

II) Per i punti del P. B. corrispondenti ai sismografi tracciamo le semirette che formano colla verticale l'angolo costante $j_{2}$ tale che sen $j_{0}=V_{1} / V_{2}$ e per il punto $N_{2}$ del contatto fra il primo e il secondo strato di profondità $h_{n 2}$ nota, o supposta nota, tracciamo la parallela la quale incontri il P. B. nel punto $P_{2}$ e quindi determiniamo il valore da attribuire al delay time dello scoppio

$$
\tau_{s 2}=\left(\tau_{i: 2}-\frac{X_{n 2}}{V_{2}}\right)-\tau_{n 2},
$$

essendo $T_{n 3}$ ed $X_{n 2}$ il tempo $T$ e la distanza $X$ corrispondenti a $P_{2}$ nella dromocrona del secondo strato e

$$
\tau_{n 2}=\frac{h_{n 2}}{V_{2}} \sqrt{\left(\frac{V_{2}}{V_{1}}\right)^{2}-1}
$$

il delay time di $P_{2}$;
III) Calcoliamo nella riga successiva a quella di $T_{i_{2}}$ i valori del delay time $\tau_{g 2}$ dei diversi sismografi sottraendo ai valori del tempo intercetto $T_{t 2}$ quello del delay time dello scoppio $\tau_{s 2}$, costante e, nella riga sottostante, calcoliamo i valori delle profondità $h_{g 2}$ dei punti del contatto del primo e del secondo strato ove i raggi si rifrangono ai diversi sismografi mediante la

$$
h_{\delta=}=\tau_{\delta 2} \frac{V_{2}}{\sqrt{\left(\frac{V_{2}}{V_{1}}\right)^{2}-1}},
$$

come si vede il fattore di $\tau_{g 2}$ è costante;

IV) Segniamo sul disegno i detti punti di rifrazione nei punti d'intersezione fra le semirette per i sismograf tracciate in II) e le linee orizzontali colle profondità calcolate le quali non debbono essere tracciate se i disegni sono su carta millimetrata: la spezzata congiungente $\mathrm{i}$ diversi punti è il profilo cercato del contatto fra il primo e il secondo strato;

V) Disponendo delle dromocrone relative a diverse stazioni di scoppio si ripeterà la costruzione per ognuna e quindi si medieranno i risultati considerando le diverse attendibilità e tenendo presente che, come pel metodo degli angoli d'emergenza, si dovranno rifare le compensazioni quando varierà la quota di partenza e che al variare della quota di partenza i profili muteranno le quote delle stesse quantità ma conserveranno immutate e sovrapponibili, le loro forme: anche in questo caso conviene sempre controllare l'esattezza della ricostruzione col calcolo diretto dei tempi e dei percorsi;

VI) Per la ricostruzione del terzo strato operiamo nel seguente modo, utilizzando il modulo coi dati corrispondenti: a) calcoliamo i rapporti $X / V_{3}$ e i valori del tempo intercetto $T_{i 3}=T_{r}-X / V_{3}$ esattamente come indicato in I) per il secondo strato, $\beta$ ) per i punti del P. B. corrispondenti ai sismografi tracciamo le semirette che formano colla verticale l'angolo $i_{13}$ tale che sen $i_{13}=V_{1} / V_{3}$ e dai punti d'incontro col contatto fra il primo e il secondo strato tracciamo le semirette clie formano colla verticale l'angolo $j_{3}$ tale che $\operatorname{sen} j_{3}=V_{2} / V_{3}$, 
quindi tracciamo per il punto $N_{3}$ di profondità $h_{n 3}$ nota, o supposta nota, le parallele alle rette adiacenti fino a incontrare in $P_{3}$ il P. B. e determiniamo il valore del delay time dello scoppio ponendo

$$
\tau_{s 3}=\left(T_{n 3}-\frac{X_{n 3}}{V_{3}}\right)-\tau_{n 3},
$$

ove ${ }^{\prime}{ }_{n 3}$ ed $X_{n 3}$ siano il tempo e la distanza corrispondente a $P_{3}$ nella dromocrona e $\tau_{n 3}$ Il delay time dello stesso $P_{3}$,

$$
\begin{gathered}
\tau_{n 3}=\frac{h_{n 2}^{\prime}}{V_{3}} \sqrt{\left(\frac{V_{i}}{\bar{V}_{1}}\right)^{2}-1}+ \\
+\frac{h_{n 3}-h_{n 2}}{V_{3}} \sqrt{\left(\frac{V_{3}}{V_{2}}\right)^{2}-1},
\end{gathered}
$$

$h_{n 2}^{\prime}$ profondità del contatto fra il primo e il secondo strato in corrispondenza al punto di rifrazione del raggio per $N_{3}, \gamma$ ) calcoliamo i valori del delay time $\tau_{23}$ dei diversi sismografi sottraendo ai valori del tempo intercetto calcolati in $\alpha$ ) quello del delay time dello scoppio $\tau_{s 3}$ e infine calcoliamo le profondità dei punti del contatto fra Il secondo e il terzo strato di rifrazione dei raggi per $i$ diversi sismografi ponendo

$$
\begin{gathered}
h_{\sigma_{2}}=\tau_{\sigma} \frac{V_{3}}{\sqrt{\left(\frac{V_{3}}{V_{2}}\right)^{2}-1}}- \\
-h_{g 2}^{\prime} \frac{\sqrt{\left(\frac{V_{3}}{V_{1}}\right)^{2}-1-\sqrt{\left(\frac{\left.V_{3}\right)^{2}}{\nabla_{2}}\right)^{2}-1}}}{\sqrt{\left(\frac{V_{3}}{V_{2}}\right)^{2}-1}},
\end{gathered}
$$

ove $h_{\text {g2 }}^{\prime}$ profondità del punto di rifrazione dei raggi al contatto fra il primo e il secondo strato (notiamo che $\mathrm{i}$ fattori di $\tau_{g 3}$ e $h_{g_{2}}$ sono supposti costanti): per agevolare $i$ calcoli converrà segnare nel modulo, alle righe seguenti quella di $\tau_{g 3}$, successivamente i valori che risultano per il primo termine della [48], quelli delle profondità $h_{g_{2}}^{\prime}$ che si rilevano dai disegni, quelli che si calcoleranno per il secondo termine e infine quelli di $h_{g 3}$, differenze fra il primo e ll secondo termine, $\delta$ ) segniamo sul disegno i punti di rifrazione nei punti d'intersezione fra le semirette che si dipartono dal contatto fra il primo e il secondo strato tracciate come in $\beta$ ) e le linee orizzontali colle profondità calcolate, congiungiamo con una spezzata continua i diversi punti e quindi completiamo le elaborazioni operando nello stesso modo indicato per il contatto fra il primo e il secondo strato in V);

VII) Per la ricostruzione del quarto strato operiamo come per quella del terzo tenendo conto che: $A$ ) le semirette per i sismografi devono avere l'inclinazione $i_{14}$ rispetto alla verticale tale che sen $i_{14}=$ $=V_{1} / V_{4}$, quelle dai punti d'incontro col contatto fra il primo e il secondo strato la inclinazione $i_{24}$ tale che sen $i_{24}=V_{2} / V_{4} \mathrm{e}$ quelle dai punti d'incontro col contatto fra il secondo e il terzo strato le semirette d'inclinazione $i_{34}$ tale che sen $i_{34}=V_{3} / V_{4}$, $B$ ) il delay time relativo al punto di profondità nota $N_{4}$ si calcola ponendo

$$
\begin{aligned}
\tau_{n 4} & =\frac{k_{n_{2}}^{\prime}}{V_{4}} \sqrt{\left(\frac{V_{4}}{V_{1}}\right)^{2}-1}+ \\
& +\frac{k^{\prime}{ }_{n 3}-k_{n_{3}}^{\prime}}{V_{4}} / \sqrt{\left(\frac{V_{1}}{V_{2}}\right)^{2}-1}+ \\
& +\frac{h_{n 4}-k_{n_{3}}^{\prime}}{V_{4}} \sqrt{\left(\frac{V_{4}}{V_{3}}\right)^{2}-1}
\end{aligned}
$$

essendo $k^{\prime}{ }_{n 2}, k_{n 3}^{\prime}$ e $h_{n s}$ le profondità rispettivamente dei punti di rifrazione del raggio per $N_{4}$ al contatto fra il primo e il secondo strato, fra il secondo e il terzo strato e fra il terzo e il quarto strato, $C$ ) le profondità $h_{g 4}$ dei punti di rifrazione al contatto fra il terzo e il quarto strato dei raggi per i sismografi si calcolano applicando la

$$
\begin{aligned}
& h_{\mathrm{gn}}=\tau_{\mathrm{s}} \frac{V_{4}}{\sqrt{\left(\frac{V_{4}}{V_{3}}\right)^{2}-1}}- \\
& -k_{n 3}^{\prime} \frac{\sqrt{\left(\frac{V_{4}}{V_{2}}\right)^{2}-1-\sqrt{\left(\frac{V_{4}}{V_{3}}\right)^{2}-1}}}{\sqrt{\left(\frac{V_{4}}{\boldsymbol{V}_{3}}\right)^{2}-1}}- \\
& -z_{n+\ldots}^{\prime} \frac{V^{\prime}\left(\frac{V_{1}}{V_{1}}\right)^{2}-1-\sqrt{\left(\frac{V_{4}}{V_{2}}\right)^{2}-1}}{\sqrt{\left(\frac{V_{4}}{V_{\varepsilon}}\right)^{2}-1}} ;
\end{aligned}
$$


per la ricostruzione del quinto strato e degli strati successivi si opererà in modo analogo.

Facciamo osservare infine: 1) che il valore del tempo intercetto calcolato secondo la [40] è generalmente diverso da quello che risulta dall'intersezione delle dromocrone coll'asse dei tempi e che perciò è da considerare erroneo il criterio di sostituire quest'ultimo al primo, come qualche volta è stato fatto, 2) che il metodo puó anche essere applicato, almeno teoricamente, quando i punti di profondità nota sono in corrispondenza ai punti di scoppio potendosi in tali casi determinare direttamente i delay times da attribuire agli scoppi stessi: è evidente però che in tali casi: a) si ammette implicitamente che gli strati siano continui per tutto l'intervallo fra gli scoppi e i sismografi, $b$ ) sui valori delle profondità risultanti in corrispondenza ai sismografi si assommano tutti gli errori sulle velocità degli strati, sempre molto sensibili (errori che entrano attraverso il calcolo del tempo intercetto).

\section{8. - Rilievo per archi.}

La particolarità di questo metodo sta nel fatto che i sismografi vengono disposti equidistanti dalla stazione di scoppio per modo che risulta costante il tempo intercetto e quindi ogni variazione dei tempi rilevati è da attribuire esclusivamente alla variazione delle quote degli strati rifrangenti, quando gli strati stessi sono continui.

In tali condizioni di rilievo, ammesso che il delay time relativo allo scoppio sia sensibilmente costante rispetto all'azimut dei sismografi (e quindi le pendenze degli strati in corrispondenza allo scoppio stesso siano piccole) le profondità del secondo strato sono date per la [43] dalla

$$
h_{g 2}=h_{112}+\frac{V_{2}}{1 /\left(\frac{V_{2}}{V_{1}}\right)^{2}-1} \Delta T,
$$

essendo $h_{n 2}$ la profondità in corrispondenza ad un punto $P_{2}$ del profilo dei sismografi nota, o supposta nota, e $\Delta T$ le differenze dei tempi dei vari sismografi per rispetto al tempo corrispondente a $P_{2}$ : natural- mente tali profondità si intendono riferite ai punti clie stanno sulle congiungenti colla stazione di scoppio spostati dell'offset verso la stazione di scoppio stessa.

Dalla [51] otteniamo la pendenza del secondo strato nella direzione del profilo dei sismografi ponendo

$$
\operatorname{tg} \alpha_{2}^{\prime}=\frac{V_{2}}{\sqrt{\left(\frac{V_{2}}{V_{1}}\right)^{2}-1}} \frac{1}{W_{2}^{\prime}},
$$

ove $W^{\prime}{ }_{2}$ la velocità apparente lungo l'arco.

Praticamente operiamo nel seguente modo, utilizzando il solito modulo di calcolo:

1) Calcoliamo i tempi ridotti $\operatorname{Tr}$ nello stesso modo indicato per i profili disposti allineati collo scoppio e quindi, nelle righe seguenti quella dei tempi $T_{r}$, calcoliamo: a) le differenze $\Delta o$ fra le distanze $X$ della prima riga (non ridotte di $\delta X$ ) e quella media $\varrho^{m}$ dei sismografi del profilo dal punto di scoppio (non dal centro della stazione), $b$ ) i rapporti $\delta T_{\varrho}=\Delta \varrho / V_{2}$, essendo $V_{2}$ la velocità vera del secondo strato, $c$ ) le differenze $T_{\varrho}=T_{r}-\delta T_{o}$.

2) Calcoliamo il valore di $T_{o}$ anche per il punto $P_{2}$ cui corrisponde la profondità $h_{n_{2}}$ nota, calcoliamo le differenze $\Delta T$ fra $\mathrm{i}$ tempi $T_{o}$ relativi ai diversi sismografi e al punto $P_{2}$ e applicando la [51] calcoliamo le profondità $h_{\mathrm{g} 2}$ relative ai sismografi;

3) Calcoliamo gli offsets $\lambda_{g 2}$ mediante la formula:

$$
\lambda_{g 2}=h_{g 2} \operatorname{tg} j_{2}=\frac{h_{\mathrm{g} 2}}{\sqrt{\left(\frac{V_{2}}{V_{1}}\right)^{2}-1}} ;
$$

4) Riportiamo sulle congiungenti dei sismografi al punto di scoppio, a partire dai sismografi stessi, i valori degli offsets $\lambda_{\sigma_{2}}$ calcolati ed in corrispondenza ai punti che risultano individuati segniamo $i$ valori delle profondità $h_{g_{2}}$ corrispondenti;

5) Per ogni stazione di scoppio evidentemente risulteranno dei valori diversi delle profondità e degli offsets in quanto i raggi registrati dai sismografi saranno relativi a direzioni diverse: $\mathrm{i}$ risultati delle diverse stazioni di scoppio si potranno quindi mediare soltanto tenendo conto della configurazione d'insieme dello strato: variando 
le profondità di partenza di una quantità $\Delta h$ tutte le profondità calcolate risulteranno variate dalla stessa quantità e gli offsets risulteranno tutti variati della quantità

$$
\Delta \lambda=\frac{\Delta h}{\sqrt{\left(\frac{V_{2}}{V_{1}}\right)^{2}-1}} ;
$$

6) Per la ricostruzione del terzo strato calcoliamo i valori di $T_{Q}$ operando nello stesso modo indicato in 1) però adottando per il calcolo di $\delta T_{\varrho}$ la velocità $V_{3}$ del terzo strato e quindi operando nel seguente modo: a) determiniamo la profondità $h_{g 2}^{\prime}$ e gli offsets $\lambda_{g_{2}}^{\prime}$ dei punti intersezione delle semirette per $\mathrm{i}$ sismografi d'inclinazione $i_{13}$ rispetto alla verticale tale per cui sen $i_{13}=$ $=V_{1} / V_{3}$ colla superficie di contatto fra Il primo e il secondo strato quale risulta dalia planimetria costruita colle elaborazioni precedenti (ciò che riuscirà molto semplice e in generale abbastanza preciso operando per via grafica) e calcoliamo i delay times dei punti del contatto fra il primo e il secondo strato di rifrazione dei raggi ai sismografi

$$
\tau_{g 2}^{\prime}=\frac{\frac{V_{\mathbf{3}}}{V_{1}^{2}}}{\sqrt{\left(\frac{V_{3}}{V_{1}}\right)^{2}-1}} h_{g 2}^{\prime}--\frac{1}{V_{z}}-\lambda_{g 2}^{\prime},
$$

b) calcoliamo le differenze $\Delta T$ fra $\mathrm{i}$ tempi $T_{0}$ relativi ai diversi sismogmfi e quello relativo al punto $P_{3}$ cui corrisponde il punto di rifrazione $N_{3}$ di profondità $h_{n 3}$ nota e calcoliamo le differenze $\Delta \tau^{\prime}{ }_{g}$ fra $\mathrm{i}$ valori di $\Delta T$ cosi ottenuti e quelli di $\tau_{\mathbf{g}_{2}}^{\prime}$ calcolati in precedenza, c) calcoliamo le profondità dei punti del contatto fra il secondo e il terzo strato di rifrazione dei raggi trasmessi ai sismografi ponendo, per la [51],

$$
h_{g 3}=h_{g 2}^{\prime}+\frac{V_{3}}{\sqrt{\left(\frac{V_{g}}{V_{g}}\right)^{2}-\vec{i}}} \tau_{g}^{\prime}
$$

e calcoliamo gli offsets degli stessi punti mediante la

$$
\lambda_{\mathrm{g3}}=\lambda_{\mathrm{g} 2}^{\prime} \frac{V_{\mathrm{g}}}{\left(\frac{V_{3}}{V_{2}}\right)^{2}-1} \Delta \tau_{g}^{\prime},
$$

d) riportiamo sulle congiungenti dei sismografi ai punti di scoppio, a partire dai sismografi stessi, i valori degli offsets $\lambda_{g 3}$ calcolati e in corrispondenza ai punti che risultano individuati segniamo i valori delle profondità $h_{\mathrm{g} 3}$ corrispondenti e infine procederemo alla compensazione dei risultati operando esattamente come in 5).

7) Per la ricostruzione del quarto strato calcoliamo i valori di $T_{\varrho}$ nel solito modo adottando la velocità $V_{1}$ del quarto strato e operando nel seguente modo: $A$ ) determiniamo le profondità $k_{g 2}^{\prime}$ e gli offsets $\mu_{F_{2}}^{\prime}$ dei punti intersezione delle semirette per i sismografi, di inclinazioni $i_{14}$ rispetto alla verticale tali per cui sen $i_{14}=V_{1} / V_{1}$, colla superficie di contatto fra il primo e il secondo strato, determiniamo inoltre le profondità $k_{g_{3}}^{\prime}$ e gli offsets $\mu_{g 3}^{\prime}$ delle intersezioni delle semirette per $i$ detti punti d'intersezione, inclinate degli angoli $i_{21}$ rispetto alla verticale tali che sen $i_{24}=V_{2}$ / $V_{4}$, colia superficie di contatto fra il secondo e il terzo strato e infine calcoliamo i delay times dei punti del contatto fra il secondo e il terzo strato di rifrazione dei raggi per $i$ sismografi

$$
\begin{gathered}
\tau_{g_{3}}^{\prime}=\frac{\frac{V_{4}}{V_{1}^{2}}}{\sqrt{\left(\frac{V_{4}}{V_{1}}\right)^{2}-1}} k_{g 2}^{\prime}+ \\
+-\frac{\frac{V_{4}}{V_{2}^{2}}}{\sqrt{\left(\frac{V_{4}}{V_{2}}\right)^{2}-1}}\left(k_{\mathrm{g} 3}^{\prime}-k_{g 2}^{\prime}\right)- \\
-\frac{1}{V_{2}} \mu_{g 2}^{\prime}-\frac{1}{V_{3}}\left(\mu_{g 3}^{\prime}-\mu_{R 2}^{\prime}\right),
\end{gathered}
$$

B) calcoliamo le differenze $\Delta T$ fra i tempi $T_{\varrho}$ relativi ai diversi sismografi e quello relativo a $P_{4}$ di profondità $h_{n_{4}}$ nota e calcoliamo le differenze $\Delta \tau^{\prime}{ }_{g}$ fra $\mathrm{i}$. valori di $\Delta T$ e quelli già determinati di $\left.\tau^{\prime}{ }_{g 3}, C\right)$ calcoliamo le profondità dei punti di contatto fra il terzo e il quarto strato di rifrazione dei raggi trasmessi ai sismografi ponendo

$$
h_{g 4}=k_{g 3}^{\prime}+\frac{V_{4}}{\left(\frac{V_{4}}{V_{3}}\right)^{2}-1} \Delta \tau_{g}^{\prime}
$$


e calcoliamo gli offsets degli stessi punti ponendo:

$$
\lambda_{\mathrm{g} 4}=\mu_{\mathrm{g} 3}^{\prime}+\frac{V_{4}}{\left(\frac{V_{1}}{\bar{V}_{3}}\right)^{2} 1} \Delta \tau_{g}^{\prime},
$$

D) riportiamo le profondità e gli offsets calcolati sui disegni dei rilievi ed eseguiamo la compensazione degli stessi nel solito modo.

Per la ricostruzione del quinto strato e degli strati successivi si procederà in modo analogo.

Questo metodo può essere considerato, evidentemente, come un caso particolare del metodo di Gardner: le osservazionifatte per questo, sui limiti di validità e sulle approssimazioni possibili, devono quindi ritenersi valide anche per il metodo di rilievo per archi. In più dobbiamo osservare che per quest'ultimo i raggi registrati dai sismografi sono relativi a percorsi affatto diversi e ogni eterogeneità del sottosuolo all'interno degli archi si ripercuote con una variazione del tempo intercetto dei punti degli archi stessi (senza che si abbia la possibilità di individuarne la causa) e quindi con delle strutture fittizie.

Tale sistema di rilievo può invece tornare in pratica conveniente quando, per la sicurezza dell'interpretazione dei risultati, si rivelasse la necessità di correlare direttamente gli impulsi rilevati su diverse linee, con stazioni di scoppio comuni, per i quali si ebbero discontinuità e incertezze.

\section{9. - Considerazioni sui diversi metodi.}

I metodi degli angoli d'emergenza e di Gardner differiscono sostanzialmente soltanto per il fatto che mentre per il secondo si ammettono dei raggi sempre uguali a quelli corrispondenti all'ipotesi degli strati orizzontali per il primo si ammettono dei raggi conformi alla reale configurazione degli strati.

Le diverse impostazioni dei calcoli dei due metodi comportano evidentemente delle precisioni diverse: però per strati con piccole pendenze anche il metodo di Gardner riesce di precisione sufficiente per la pratica e quindi non vi sarebbe ragione di complicare i calcoli applicando il metodo degli angoli d'emergenza. In effetto il metodo di Gardner riesce più semplice di quello degli angoli d'emergenza soltanto nel caso che si abbiano due o tutt'al più tre strati, come possiamo facilmente convincerci considerando le complicazioni che intervengono al crescere del numero degli strati nel calcolo delle profondità, da farsi colle formole [48], [50], ecc.

Per evitare queste complicazioni viene sovente consigliato di predisporre dei grafici-prontuari coi quali determinare rapidamente le profondità e gli offsets dei punti di rifrazione in funzione del delay time: ̀̀ evidente peró che grafici del genere possono essere predisposti soltanto considerando una particolare successione degli strati e quindi limitando ancora più la generalità del metodo e la precisione raggiungibile collo stesso.

Poichè quindi i vantaggi che il metodo di Gardner offre in confronto a quello degli angoli d'emergenza si riducono a qualche facilitazione di poco conto dei calcoli noi riteniamo che come regola convenga applicare il metodo degli angoli d'emergenza nei rilievi di prima esplorazione e convenga riservare l'applicazione di quello di Gardner alle zone che dai primi rilievi appaiono rispondenti alle condizioni per le quali lo stesso metodo può essere proficuamente applicato, posto che per le stesse zone interessino i rilievi di maggiore dettaglio.

In presenza di faglie entrambi i metodi sono in difetto e le quote non possono essere portate attraverso le stesse se non coi calcoli diretti dei percorsi e dei tempi relativi. Applicando il metodo di Gardner evidentemente per ogni quota così determinata si dovrà ricalcolare il delay time corrispondente onde avere il delay time da attribuire allo scoppio per il computo delle profondità relative ai sismografi successivi.

Pure per entrambi i metodi le precisioni raggiungibili diminuiscono rapidamente al crescere del numero degli strati presenti e delle profondità e gli errori commessi sulle quote di partenza si manifestano con spostamenti orizzontali degli assi delle pieghe.

Il sistema di rilievo consigliato da Gardner, in presenza di un "marker" ben caratteristico, di scoppiare a distanze costanti dai sismografi in modo da avere come primo impulso sempre le onde rifratte del marker, risparmiando il rilievo degli intervalli più 
vicini delle linee, non è meglio applicabile col metodo dallo stesso Gardner proposto piuttosto che col metodo degli angoli d'emergenza: noi abbiamo già detto però al par. 1 come un tale sistema non sia assolutamente da consigliare nei rilievi pratici neanche nelle zone già coperte da un rilievo a larghe maglie.

Riguardo al metodo di rilievo per archi è da osservare che in presenza di una faglia la quale interseca il profilo dei sismografi si hanno degli andamenti dei tempi tutt'affatto diversi da quelli che abbiamo visto al par. 13 relativi al caso dei profili dei sismografi allineati cogli scoppi: ciò principalmente per effetto delle rifrazioni laterali dei lembi delle faglie di quote diverse.

\section{0. - Compensazioni e batimetrie.}

I profili delle superfici $\varsigma_{12}, \varsigma_{13}, \ldots$ di contatto dei diversi mezzi $M_{1}, M_{2}, M_{3}, \ldots$ che costituiscono il sottosuolo ottenuti colle diverse linee di rilievo $\Lambda^{\prime}, \Lambda^{\prime \prime}, \ldots$, devono essere paragonati fra loro e compensati nelle inevitabili differenze che accusano.

Nel caso del contatto fra il primo e il secondo strato, secondo la superficie $\varsigma_{12}$, tali profili sono relativi alla sezione per la traccia sul P. B. della linea di rilievo normale in ogni punto alla superficie $\varsigma_{12}$.

Ciò posto, possiamo determinare le caratteristiche geometriche della $\varsigma_{12}$ in corrispondenza al punto $R$ d'intersezione dei profill relativi a due linee di rilievo $\Lambda^{\prime}$ e $\Lambda^{\prime \prime}$ qualunque intersecantesi in un punto $G_{0}$ nel seguente modo:

a) centro nel punto $G^{\prime}$ di $\Lambda^{\prime}$ d'intersezione colla $\Lambda^{\prime \prime}$ tracciamo l'arco di cerchio tangente al profilo di $\varsigma_{12}$ e per il punto di tangenza $R^{\prime}$ tracciamo la verticale fino a incontrare la traccia del P. B. in $R_{1}$, sia $\varrho_{2}$ Il raggio dell'arco tracciato;

$b$ ) ugualmente per la linea $\Lambda^{\prime \prime}$, centro nel punto $G^{\prime \prime}$ d'intersezione colla $\Lambda^{\prime}$ tracciamo l'arco tangente al profilo e per il punto di tangenza $R^{\prime \prime}$ tracciamo la verticale fino a incontrare la traccia del P. B. in $R_{2}$, sia $\varrho_{2}^{\prime \prime}$ il raggio dell'arco tracciato;

c) poichè i punti $R^{\prime}$ ed $R^{\prime \prime}$ devono essere coincidenti con $R$ sarà necessariamente $\varrho_{2}=\varrho_{2}^{\prime}=\varrho_{2}^{\prime \prime} ;$ d'altra parte, siccome gli stessi punti $R$ ' ed $R^{\prime \prime}$ non possono che trovarsi sulle circonferenze di raggio $\underline{o}_{2}$ e coi centri in $\kappa_{1}$ ed $R_{2}$ contenute nei piani verticali per gli stessi punti normali alle linee $\Lambda^{\prime} \mathrm{e}$ $\Lambda^{\prime \prime}$ rispettivamente, la proiezione sul P. B. $R_{o}$ di $R$ è data dall'intersezione delle normali alle linee $\Lambda^{\prime}$ e $\Lambda^{\prime \prime}$ per $R_{1}$ ed $R_{2}$, sul P. B. stesso;

d) la direzione della linea di massima pendenza della superficie $\varsigma_{12}$ è data sul P. B. dalla congiungente il punto d'incrocio $G_{o}$ colla proiezione $R_{o}$ e l'inclinazione (massima) lungo la stessa linea è data dalla

$$
a_{2}=\operatorname{arcsen} \frac{G_{n} R_{o}}{\varrho_{2}},
$$

conforme alla legge di composizione dei vettori seno delle inlienazioni di cui abbiamo detto al par. 11.

Per la compensazione della rete delle linee rilevate si possono paragonare le quote dei diversi profili per i punti d'intersezione, in accordo con quanto abbiamo detto in $c$ ).

Ogni maglia della rete rilevata deve evidentemente chiudere con "contraddizione" nulla: se questa viceversa non sarà nulia ne ripartiremo il valore tenendo conto dell'attendibilità dei dati di rilievo e, se del caso, dei controlli che risultano dalie maglie adiacenti, secondo i principi della nota teoria delle compensazioni mediate.

$\mathrm{E}$ da tenere presente che variando sensibilmente le quote di partenza dei profili questi devono essere di nuovo determinati quando viene utllizzato il metodo di elaborazione degli angoli d'emergenza, come già abbiamo fatto osservare al par. 16 .

Per la costruzione delle batimetrie delle superfici $\varsigma_{12}$ a rigor di termini sono utilizzabili soltanto i punti $R_{o}$ che risultano dagli incroci delie diverse linee di rilievo, i quali come ben si comprende sono sempre molto scarsi. Per ovviare a questo inconveniente col minimo di arbitrarietà noi riteniamo che convenga inserire delle linee fittizie fra le linee rllevate, interpolando fra queste ultime, in modo da formare una rete con maglie sufficientemente ridotte, per esempio di $400 \times 400$ metri tutte perfettamente compensate, e quindi utilizzare i punti $R_{o}^{\prime}$ che risultano dagli incroci delle linee reali e fittizie.

Per poter interpolare con precisione e sicurezza fra le quote dei punti $R_{o}$ converrà 
tracciare anche le curve continue che congiungono i punti $R_{o}$ relativi alle stesse linee, le quali non sono altro che proiezioni verticali sul P. B. delle intersezioni delle normali alla superficie $\varsigma_{12}$ di contatto fra il primo e il secondo strato abbassate dalle proiezioni delle linee sul P. B. stesso, naturalmente tenendo conto degli andamenti dei profili delle linee relative.

Le linee di livello delle batimetrie, evidentemente, in corrispondenza ai punti $R_{o}$ saranno perpendicolari alle congiungenti coi punti $G$ d'incrocio e saranno fra loro distanziate in modo conforme ai valori delle pendenze che risultano dalla [60] per la superficie $\varsigma_{12}$.

Se consideriamo i profili delle diverse linee riferiti a piani verticali la costruzione delle batimetrie riesce molto semplificata, in quanto ogni punto dei profili risulta determinato nello spazio mentre col sistema rigoroso risultano noti nello spazio soltanto quelli corrispondenti alle intersezioni dei profili delle diverse linee. Gli errori che una semplificazione del genere comporta non eccedono, in generale, quelli clie risultano per altre cause che in pratica non si riescono ad eliminare quando le inclinazioni della superficie $\varsigma_{12}$ di contatto fra il primo e il secondo strato sono minori di $10 \div 12^{\circ}$ e sempre quando viene utilizzato il metodo di Gardner, il quale, come sappiamo, ha limiti di applicazione pressochè uguali.

Le stesse costruzioni di cui sopra possono essere applicate, senza alcuna modifica, per le batimetrie delle superfici di contatto fra il secondo e il terzo strato, fra il terzo e il quarto strato, ecc., quando tali superfici sono parallele a quella di contatto fra il primo e il secondo strato.

Per il caso generale di strati comunque inclinati l'uno rispetto all'altro la costruzione rigorosa delle batimetrie è possibile soltanto per successive approssimazioni, tenendo conto, nelle ricostruzioni dei profili e nelle composizioni degli elementi, delle pendenze vieppiù approssimate che risultano per le superfici. Per la pratica però noi riteniamo che non sia consigliabile l'adozione di un simile metodo sia percliè laboriorissimo sia perchè fonte non indifferente di errori da parte degli operatori. Noi proponiamo il seguente metodo approssimato:

A) ammesso di aver costruito la batimetria della superficie $\varsigma_{12}$ di contatto fra il primo e il secondo strato nel modo che sopra abbiamo descritto, facendo centro nei punti $R^{\prime}$ ed $R^{\prime \prime}$ dei profili di $\varsigma_{12}$ delle linee $\Lambda^{\prime}$ e $\Lambda^{\prime \prime}$ tracciamo gli archi di cerchio tangenti ai profili della superficie $\varsigma_{23}$ di contatto fra il secondo e il terzo strato: siano $Q^{\prime}$ e $Q^{\prime \prime}$ i punti di tangenza rispettivamente sulle linee $\Lambda^{\prime}$ e $A^{\prime \prime}$; quindi innalziamo per gli stessi punti le verticali fino a incontrare le tracce del P. B. rispettivamente nei punti $Q_{1}$ e $Q_{2}$;

$B)$ poichè i raggi dei due cerchi tangenti al solito devono essere resi uguali fra loro dalla compensazione, operando come indicato in $c$ ) e $d$ ), determiniamo sulla planimetria del rilievo il punto d'incontro $Q_{0}$ fra le normali alle linee $\Lambda^{\prime}$ e $\Lambda^{\prime \prime}$ per i punti $Q_{1}$ e $Q_{2}$ quali risultano da $A$ ): la direzione di massima pendenza della superficie $\varsigma_{23}$ di contatto fra il secondo e il terzo strato nel punto $Q$ di proiezione $Q_{0}$ è data dalla congiungente $R_{0} Q_{0}$ e l'inclinazione (massima) lungo la stessa linea è data dalla

$$
\alpha_{3}=\operatorname{arcsen} \frac{R_{0} Q_{0}}{\varrho_{3}}
$$

essendo $\varrho_{3}$ il raggio comune dei due archi di cerchio tangenti al profilo di $\varsigma_{23}$;

$C)$ per le batimetrie delle superfici $\varsigma_{34}$ di contatto fra il terzo e il quarto strato operiamo in modo analogo, tracciando gli archi tangenti ai profili di $\varsigma_{34}$ con centro nei punti $Q_{1}$ e $Q_{2}$ e considerando per la direzione della massima pendenza la congiungente di $Q_{0}$ col punto $P_{0}$ corrispondente ai punti di tangenza; e cosi di seguito.

Per le compensazioni si opera pure in modo analogo a quello indicato per la prima planimetria, rendendo uguali i raggi degli archi tangenti tenendo conto delle attendibilità dei rilievi delle linee e, naturalmente, per la costruzione delle batimetrie converrà ancora ricorrere alle linee fittizie e agli altri artifici consigliati per quella del primo contatto. 


\section{RIASSUNTO}

Viene presentata, in forma monografica, la tecnica adottata dagli autori per la prospezione sismica a rifrazione.

Particolarmente da segnalare è l'esposizione di un nuovo metodo di elaborazione dei risultati denominato "metodo degli angoli d'emergenza ", il quale, nell'applicazione pratica, risulta molto rapido, oltrechè chiaro e preciso.

\section{$A B S T R A C T$}

The paper illustrates the methods employed by the Authors in refractive seismic prospecting.

Particular attention is drawn to the description of a new method for processing result, termed "the emergence angle method" which, in its practical application, proves as rapid as it is reliable and precise.

\section{BIBLIOGRAFIA}

Contini, C., La diffrazione nei rilievi sismici a riflessione, "Annali di Geofisica ", 6, 73-111, (1953).

GARDNER, L. W., An areal plan of mapping subsurface structure by refraction shooting, "Geophysics ", 4, 247-259, (1939).

Romagna Manoja, A., Distribution of seismic energy in stratified media, relazione letta all'XI Convegno dell'E.A.E.G. di Milano, 1956.

Silogffer, J. et Diemer, E., Rapporteur pour la construction rapide du rayon refracte, "Geophysical Prospecting ", 3, 234-239, (1955). 